Article

\title{
Petrogenesis and Geochronology of Tianshui Granites from Western Qinling Orogen, Central China: Implications for Caledonian and Indosinian Orogenies on the Asian Plate
}

\author{
Muhammad Saleem Mughal 1,2, Chengjun Zhang 1,*, Amjad Hussain ${ }^{3}$, Hafiz Ur Rehman 4 (D), \\ Dingding Du ${ }^{5}{ }^{\mathbb{D}}$, Mirza Shahid Baig ${ }^{2}$, Muhammad Basharat ${ }^{2}$, Jingya Zhang ${ }^{1}$, Qi Zheng ${ }^{1}$ and \\ Syed Asim Hussain ${ }^{6}$ (D) \\ 1 School of Earth Sciences \& Key Laboratory of Mineral Resources of Gansu Province, Lanzhou University, \\ Lanzhou 730000, China; muhammadsaleem152@yahoo.com (M.S.M.); jyzhang17@lzu.edu.cn (J.Z.); \\ zhengq18@lzu.edu.cn (Q.Z.) \\ 2 Institute of Geology, University of Azad Jammu and Kashmir, Muzaffarabad 13100, Pakistan; \\ drshahidbaig@gmail.com (M.S.B.); basharatgeo@yahoo.com (M.B.) \\ 3 Faculty of Earth Resources, China University of Geoscience, Wuhan 430074, China; \\ amjad.khoshhall@gamil.com \\ 4 Graduate School of Sciences and Engineering, Kagoshima University, Kagoshima 890-0065, Japan; \\ hafiz@sci.kagoshima-u.ac.jp \\ 5 School of Earth Sciences, East China University of Technology, Nanchang 330013, China; dudd15@lzu.edu.cn \\ 6 Qinghai Institute of Salt Lakes, Chinese Academy of Science, Xining 810008, China; s.asim_110@yahoo.com \\ * Correspondence: cjzhang@lzu.edu.cn
}

Received: 15 April 2020; Accepted: 25 May 2020; Published: 2 June 2020

\begin{abstract}
The precise timing, petrogenesis, and geodynamic significance of three granitoid bodies (Beidao granite, Caochuanpu granite, Yuanlongzhen granite, and the Roche type rock) of the Tianshui area in the Western Qinling Orogen, central China, are poorly constrained. We performed an integrated study of petrology, geochemistry, and zircon $\mathrm{U}-\mathrm{Pb}$ dating to constrain their genesis and tectonic implication. Petrographic investigation of the granites shows that the rocks are mainly monzogranites. The $\mathrm{Al}$ saturation index $\left(\mathrm{A} / \mathrm{CNK}\right.$ versus $\left.\mathrm{SiO}_{2}\right)$ of the granitoid samples indicates meta-aluminous to peraluminous I-type granites. Their magmas were likely generated by the partial melting of igneous protoliths during the syn-collisional tectonic regime. Rare-earth-elements data further support their origin from a magma that was formed by the partial melting of lower continental crust. The Beidao, Caochuanpu, and Yuanlongzhen granites yielded U-Pb zircon weighted mean ages of $417 \pm 5 \mathrm{Ma}$, $216 \pm 3 \mathrm{Ma}$, and $219 \pm 3 \mathrm{Ma}$, respectively. This study shows that the Beidao granite possibly formed in syn- to post-collision tectonic settings due to the subduction of the Proto-Tethys under the North China Block, and can be linked to the generally reported Caledonian orogeny (440-400 Ma) in the western segment of the North Qinling belt, whereas Yuanlongzhen and Caochuanpu granites can be linked to the widely known Indosinian orogeny (255-210 Ma). These granitoids formed due to the subduction of the oceanic lithospheres of the Proto-Tethyan Qinling and Paleo-Tethyan Qinling. The Roche type rock, tourmaline-rich, was possibly formed from the hydrothermal fluids as indicated by the higher concentrations of boron leftover during the late-stages of magmatic crystallization of the granites.
\end{abstract}

Keywords: Tianshui granites; monzogranites; geochemistry; U-Pb zircon ages; partial melting; subduction of oceanic lithospheres 


\section{Introduction}

The Qinling Orogenic Belt (QOB) separates the North China Block (NCB) from the South China Block (SCB) [1-6] and is considered to have formed by the long-term convergence between the NCB and SCB (Figure 1a) [2,4,7-9]. The QOB is a composite structure that has undergone a complex history of rifting, oceanic subduction, continent-continent collision, and intracontinental orogenic deformation [10]. It has experienced four tectono-thermal events during the Neoproterozoic, Paleozoic, Early, and Late Mesozoic, respectively, equivalent to four periods of granitoid intrusions [11]. Therefore, the QOB is significant for the investigation of the connection between orogenic processes and granitoid magmatism.

Previous researchers investigated the developments of major regional structural elements, as well as the composition and ages of a variety of rocks (i.e., ophiolitic mélanges and associated extrusive rocks, mafic-acidic intrusions, and basement and cover sequences) from the orogen. Two suture zones marked by ophiolite complexes (the Shangdan Suture Zone and the Mianlüe Suture Zone, Figure 1a) are related to orogenic events that occurred during the Devonian and Late Triassic, respectively [2,10,12-14]. It is separated into two narrow segments, i.e., the Eastern Qinling Orogenic Belt (EQOB) and the Western Qinling Orogenic Belt (WQOB) [15]. The WQOB is known as the "Golden Triangle" region (Sichuan-Shanxi-Gansu) and is the second-largest Carlin-type and Carlin-like gold province in the world $[16,17]$.

A number of hypotheses on the origin of the Paleozoic and Mesozoic granitoids have been developed in past publications [18-28]. The Paleozoic (ca. 462 to $418 \mathrm{Ma}$ ) Huichizi granite (Figure 1a, located to the southeast of the study area but within the same geological unit) was interpreted to have formed in a syn-collisional setting between the NCB and the Qinling microcontinent that was associated with the Caledonian Orogeny by previous authors $[18,19]$. An isotopic ( $\mathrm{Pb}-\mathrm{Sr}-\mathrm{Nd})$ compositional contrast between the Indosinian granitoids of the EQOB and the WQOB reveals that their crustal basements are different from each other [15]. Previous studies [20,21] conducted on the Mesozoic granitoids of Zhongchuan (232 \pm 1 to $236 \pm 2 \mathrm{Ma}$ ) and Mishuling (213 $\pm 3 \mathrm{Ma}$ ) in the WQOB (Figure 1b) suggested that their magmas were formed by partial melting of Late Mesoproterozoic crust and subcontinental lithosphere mantle and/or mixing between the felsic and mafic magmas. In contrast, Wang et al. [22] geochemically investigated the relatively younger granitoids from the nearby areas (Changba $213 \pm 2 \mathrm{Ma}$ and Huangzhuguan $214 \pm 1 \mathrm{Ma}$ and $213 \pm 3 \mathrm{Ma}$ ) in the WQOB (Figure 1b) and suggested that they were derived by anatexis of the continental crust during the Late Triassic. 

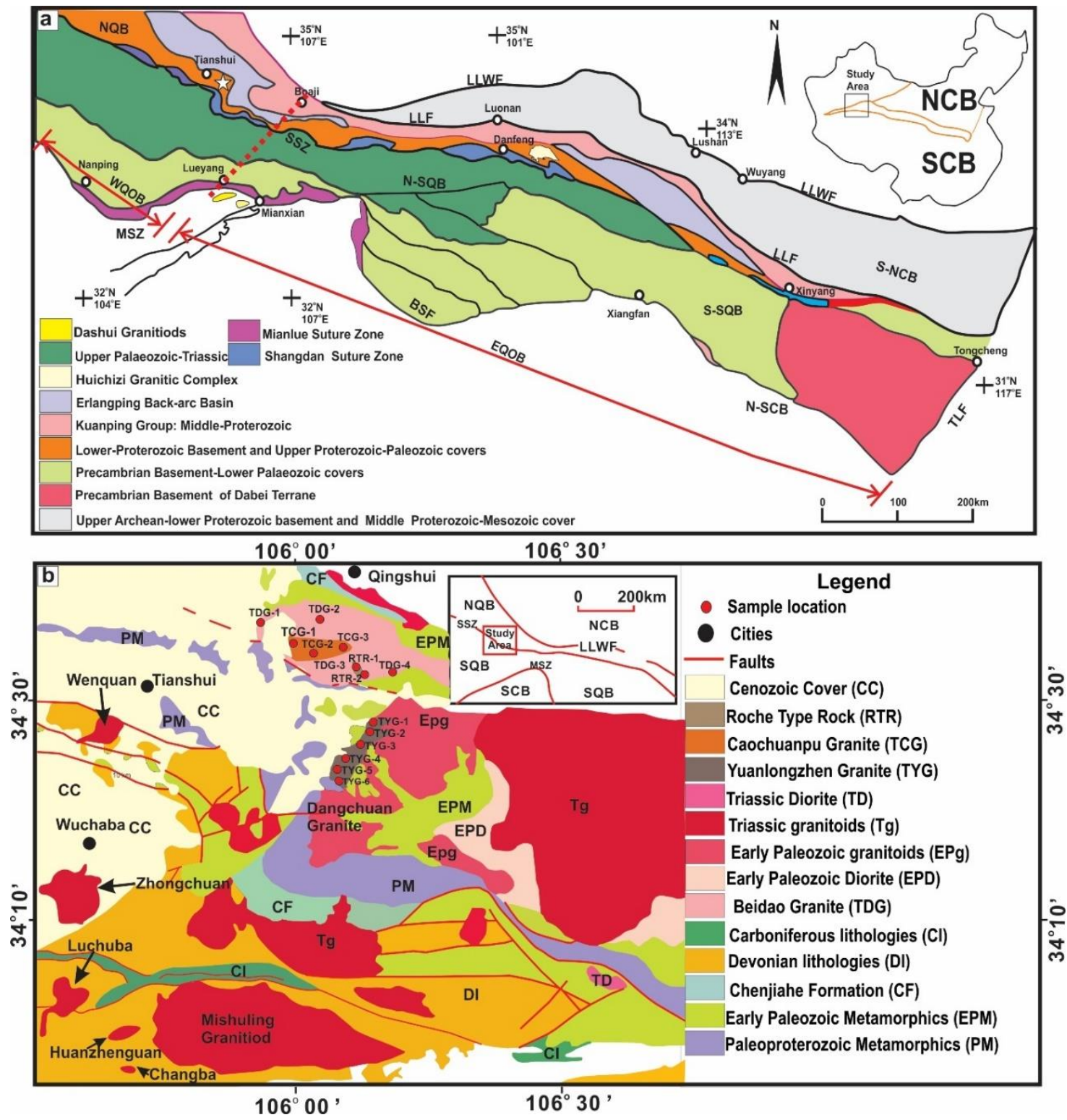

Figure 1. (a) Simplified geological and tectonic framework of the Qinling Orogenic Belt after [3]. Study area is located in the inset given at the top right corner where the square marks the study area. Granitoid rocks used in this study are located near Tianshui, marked by filled star. Red dashed line indicates the boundary between the Eastern and Western Qinling Orogenic Belt. (b) Geological map of the Tianshui area (after [23]). Small red circles indicate the sample locations that were used in this study. Abbreviations used on the map are: NCB = North China Block, SCB = South China Block, LLWF $=$ Linbao-Lusahan-Wuyang fault, LLF = Luonan-Luanchuan fault, BSF = Bashan arc-shape thrust fault, TLF = the Tanlu fault, SSZ = Shangdan Suture Zone, MSZ = Mianlüe Suture Zone, NQB = North Qinling Belt, SQB = South Qinling Belt.

According to Han et al. [24], the Dashui granitoids (214 $\pm 3 \mathrm{Ma}, 215 \pm 2$ and $210 \pm 2 \mathrm{Ma})$ in the WQOB (Figure 1a) were derived mainly from the partial melting of underlying oceanic lithosphere, and probably assimilated immature sediments during the upward migration of magma. Li et al. [25], based on published data of the Triassic granitoids from the QOB, suggested that granitoids north of the Mianlüe suture were associated with the northward subduction of the oceanic lithosphere during 248-200 Ma. Wang et al. [26] concluded that the Late Mesozoic granitoids in the QOB possibly have an identical petrogenetic mechanism to the granitoids found in the western Pacific margin, suggesting they experienced similar tectonic settings and formed by the subduction of the Pacific plate under the NCB. 
Kong et al. [27] studied the Luchuba ( $211 \pm 1 \mathrm{Ma})$ and Wuchaba $(219 \pm 1 \mathrm{Ma})$ granitoids in the WQOB and inferred that the parental magma of these granitoids was derived by the partial melting of Mianlüe oceanic crust during the collision between the NCB and the Yangtze Block. Fractional crystallization associated with crustal assimilation of parental magmas plays an important role in the generation of granitoids. Based on regional geology and geochemical results, Zhu et al. [28] inferred that the Wenquan Mo-bearing granitoids formed during the Triassic collision between the South Qinling and the SCB. Chen et al. [6] performed geochronological and geochemical studies of the magmatic rocks and Yangmugou Mo deposit in the South Qinling. The above authors proposed that their magmas formed during the collision between the NCB and the Yangtze Block in the Late Triassic, under local stress relaxation or in a local extensional environment.

All these studies were specific to the region and were carried out at reconnaissance levels only. Detailed investigation of granitoids of the Tianshui area (e.g., Beidao, Caochuanpu, and Yuanlongzhen), based on petrology and geochronological (U-Pb zircon) analysis, has not been undertaken so far. The WQOB is significant for understanding the tectonic evolution of the Asian Plate, but little is known because of the complex deformation and tectonic variation of the region. The study of granitoids is essential, not only to understand their origin but also to unravel their geodynamic and tectonic evolution. The purpose of this study thus was to petrogenetically characterize the Tianshui granitoids of the WQOB, using geochemical and geochronological methods, and to understand their geodynamic evolution.

\section{Geological and Tectonic Setting of the Area}

The Qinling Orogenic Belt is situated between the NCB and the SCB. It connects with the Dabie and the Tongbai Mountains in the east and Kunlun and Qilian Mountains in the west. It is also known as the Central China Orogen (Figure 1a). The QOB, from south to north, can be divided into four units separated by thrust faults or ductile shear zones. These units are the northern margin of the SCB (N-SCB), the South Qinling belt (SQB), the North Qinling belt (NQB), and the southern margin of the NCB (S-NCB) (Figure 1a) $[13,29,30]$. The western part of the Qinling Mountains is located in the northeast of the Qinghai-Tibet Plateau. The structural pattern of the WQOB is due to the three-dimensional compressional forces of the ancient Asian Ocean in the north, the Paleo-Tethys Ocean in the southwest and the Marginal-Pacific Ocean in the southeast [31,32] interpreted that this complex tectonic feature in the area is responsible for the formation of Cenozoic fault-bounded basins and deep faults along the northwest to southeast (NW-SE) direction. Late Paleozoic flysches, mainly Devonian sedimentary rocks, and Triassic-Jurassic granite outcrops are common in the area. The Paleo-Tethys ophiolites were exposed on the northern edge of the WQOB [2]. Han et al. [33], based on paleomagnetic and geological data, inferred that the Paleo-Tethys Ocean closed during the Triassic in the QOB. The Cenozoic alkaline volcanic rocks in the WQOB are thinly spread in the Xihe, Lixian, and Dangchang counties of the Gansu Province [34,35]. The alkaline volcanic zones include tens of ultrabasic volcanic pipes or vents developed in the Cenozoic Tianshui-Lixian fault basin [36], that coexist with carbonatites. The volcanic rocks in the area have eruption ages of $23 \mathrm{Ma}, 18.9 \mathrm{Ma}, 7.9 \mathrm{Ma}$, and 7.1 Ma, respectively [36,37].

The NQB is mainly comprised of Qinling Complex (QC) that includes meta-sedimentary and meta-volcanic rocks of the Neoproterozoic and Lower Paleozoic age comprising the Qinling and Erlangping groups [2,3]. The Qinling Group is predominantly comprised of gneisses, marble, amphibolites, and subordinate metapelites [2]. All these rocks were intruded by granitoids of the Neoproterozoic and Silurian-Devonian age [38]. The Qinling Group is considered as crystalline basement rocks of the Precambrian and lies in the inner core of the NQB. It contains the oldest gneisses (2267-2172 Ma) and meta-tholeiite amphibolites (1987 Ma) [8,39]. Moreover, some other gneisses in the Qinling Group have protolith ages of ca. 1000-800 Ma [40] and metamorphic ages of ca. 996-793 Ma [41]. The Erlangping Group is composed of an ophiolitic unit, clastic sedimentary successions, and carbonates (after [3,21]). The ophiolitic unit is predominantly comprised of gabbro, 
basalt, marble with some radiolarian chert, and thin ultramafic rock. Moreover, the zircon U-Pb ages of 463-474 Ma are reported from pillow lavas, diabase, and gabbro in the ophiolitic mélange [42]. The clastic rocks comprised of low-amphibolite facies mica-quartz schist, meta-quartzose arkose, and meta-siltstone with volcanic rocks [43]. The youngest detrital zircon age of ca. $500 \mathrm{Ma}$ was reported from the meta-clastic sedimentary rocks [44]. The Beidao granite intrudes the Qinling Group that cuts the regional metamorphic schistosity. The Yuanlongzhen granite intrudes both the Qinling Group and the Early Paleozoic granites. The Caochuanpu granite has intrusive contact with the Beidao granite.

\section{Materials and Methods}

Fifteen representative rock samples were collected from the Tianshui area in the WQOB (Figure 1b). Out of these, 4 samples were collected from the Beidao granite (TDG-1, TDG-2, TDG-3, and TDG-4), 3 from the Caochuanpu granite (TCG-1, TCG-2 and TCG-3), 6 from the Yuanlongzhen granite (TYG-1, TYG-2, TYG-3, TYG-4, TYG-5 and TYG-6), and 2 from the Roche type rock (RTR-1 and RTR-2) (see sample location in Figure 1b). All the samples were petrographically investigated under an optical microscope and the modal abundance of mineral phases is shown in Table 1.

Major elements from the representative rock samples were determined through $\mathrm{X}$-ray fluorescence (XRF) spectroscopy. Samples were dried and crushed to finer than 200 mesh $(75 \mu \mathrm{m}$ or less). About $4 \mathrm{~g}$ of the sample plus boric acid were weighed and pressed into a round cake $(4 \mathrm{~cm}$ diameter and $8 \mathrm{~mm}$ thick). Compressed samples were measured for major and trace elements using the Panalytical (Magix PW2403, The Netherlands) XRF at an operating temperature of $20 \pm 0.05^{\circ} \mathrm{C}$ with an analytical error of about $2 \%$. All the samples were analyzed in the Ministry of Education's Key Laboratory of Resources and Environment of Lanzhou University, China. The results are shown in Table 2.

Trace and rare earth element (REE) contents were measured by Elan 6100 inductively coupled plasma-mass spectrometer (ICP-MS) at the Key Laboratory of Crust-Mantle Materials and Environments of Chinese Academy of Sciences (CAS) at the University of Science and Technology of China (USTC). The analytical conditions and procedures were the same as described in Gao [45]. Prior to analyses, about $50 \mathrm{mg}$ rock powder of the sample was completely digested by acid-dilution (mixed acid of $2 \mathrm{~mL}$ $10 \mathrm{M} \mathrm{HNO}_{3}$ and $2 \mathrm{~mL} 20 \mathrm{M} \mathrm{HF}$ ) in a high-pressure PTFE bomb. The bombs were steel-jacketed and placed in the oven at $190^{\circ} \mathrm{C}$ for $72 \mathrm{~h}$. Petrological standard of GSP-2 was chosen for calibrating the elemental concentration. The analytical precision varies between $\sim 5 \%$ and $10 \%$. The results are shown in Table 2.

Zircon U-Pb dating and trace elements in the same dated-spot were carried out on three samples (TDG-1, TCG-2, and TYG-1). Zircon separation was performed by Langfang Integrity Geological Services Ltd. using the conventional (heavy liquid, followed by handpicking) separation method. Internal zircon morphology was investigated by cathodoluminescence $(\mathrm{CL})$ prior to the $\mathrm{U}-\mathrm{Pb}$ isotope analysis. The U-Pb dating was conducted at the Key Laboratory of Crust-Mantle Materials and Environments of CAS at the USTC using the ArF excimer laser ablation system (193-nm wavelength), connected to an Agilent 7700E ICP-MS. The parameters used are energy density of $10 \mathrm{~J} \mathrm{~cm}^{-2}$, ablation spot size was $32 \mu \mathrm{m}$, average power output of about $4 \mathrm{~W}$, repetition rate of $10 \mathrm{~Hz}$ (sometimes $6 \mathrm{~Hz}$ wherever necessary), and a laser pulse of 400 . Helium was used as the carrier gas in the GeoLas system to increase the transport efficiency of the ablated material. Each analysis took approximately $24 \mathrm{~s}$ background acquisition followed by $40 \mathrm{~s}$ for data acquisition from the sample. The SRM610 and Zircon 91500 were used as external standards for the elemental and U-Pb isotope ratio calibrations, respectively.

For detailed analysis procedures and instrument parameters refer to $\mathrm{Gu}$ et al. [46] and Sun et al. [46]. Analytical precision and results for the Zircon 91500 (ca. $1062 \pm 5 \mathrm{Ma}$ ) were consistent with the reported by Wiedenbeck et al. [47]. The U/Pb processing software was LaDating (version 1.8), and the quantitative calibration of the $\mathrm{Pb}$ isotope dating was performed by Common $\mathrm{Pb}$ correction (ComPbCor \#3_18) [48]. The concordia plots and weighted average age calculations were carried out by Isoplot 4.15 [49]. The zircon $\mathrm{U}-\mathrm{Pb}$ results are listed in Table 3 and the trace element compositions of analyzed zircons are shown in Table 4. 
Table 1. The modal mineralogical composition of granites and Roche type rock in the Western Qinling Orogenic Belt (WQOB).

\begin{tabular}{|c|c|c|c|c|c|c|c|c|c|c|c|c|c|c|c|}
\hline \multirow{2}{*}{$\begin{array}{l}\text { Modal Mineralogical } \\
\text { Composition }\end{array}$} & \multicolumn{4}{|c|}{ Beidao Granite } & \multicolumn{3}{|c|}{ Caochuanpu Granite } & \multicolumn{6}{|c|}{ Yuanlongzhen Granite } & \multicolumn{2}{|c|}{ Roche Type Rock } \\
\hline & TDG-1 & TDG-2 & TDG-3 & TDG-4 & TCG-1 & TCG-2 & TCG-3 & TYG-1 & TYG-2 & TYG-3 & TYG-4 & TYG-5 & TYG-6 & RTR-1 & RTR-2 \\
\hline $\mathrm{Qz}$ & 32 & 35 & 35 & 27 & 25 & 28 & 30 & 30 & 31 & 30 & 29 & 32 & 28 & 60 & 66 \\
\hline Pl & 30 & 25 & 24 & 30 & 30 & 27 & 24 & 28 & 30 & 25 & 26 & 23 & 24 & - & - \\
\hline \multirow{3}{*}{ Afs } & 5 & 3 & 6 & 5 & 40 & 35 & 37 & 22 & 24 & 25 & 21 & 27 & 25 & - & - \\
\hline & 15 & 18 & 12 & 15 & - & - & - & 8 & 6 & 8 & 13 & 7 & 10 & - & - \\
\hline & 8 & 9 & 7 & 13 & - & - & - & - & - & - & - & - & - & - & - \\
\hline $\mathrm{Bt}$ & 5 & 2 & 5 & 5 & 1 & 4 & 4 & 5 & 2 & 4 & 3 & 3 & 4 & - & - \\
\hline Ms & 0.5 & 1 & 1 & 1 & - & - & - & - & - & 1 & - & 1 & 1 & 3 & 1 \\
\hline $\mathrm{Zrn}, \mathrm{Ap}, \mathrm{Rt}, \mathrm{Tnt}$ & 2 & 2 & 2 & 1 & 2 & 1 & 1 & 2 & 2 & 1 & 2 & 1 & 1 & 1 & 1 \\
\hline Ser & 2 & 4 & 6 & 2 & 1 & 3 & 1 & 3 & 3 & 5 & 3 & 5 & 5 & - & - \\
\hline Tur & - & - & - & - & - & - & - & - & - & - & - & - & - & 36 & 32 \\
\hline Opq & 1 & 1 & 2 & 1 & 1 & 2 & 3 & 2 & 2 & 1 & 3 & 1 & 2 & - & - \\
\hline Total & 100 & 100 & 100 & 100 & 100 & 100 & 100 & 100 & 100 & 100 & 100 & 100 & 100 & 100 & 100 \\
\hline
\end{tabular}

Abbreviations shown in the table are: Afs: alkali feldspar, Ap: fluorapatite, Bt: biotite, Mc: microcline, Mc prt: microcline perthite, Ms: muscovite, Myr: myrmekite, Opq: opaque minerals,

Pl: plagioclase, Prt: perthite, Qz: quartz, Rt: rutile, Ser: sericite, Tnt: titanite, Tur: tourmaline, and Zrn: zircon. The Roche type rock contains no feldspar.

Table 2. Major, trace, and rare earth elements contents of the Tianshui granitoids.

\begin{tabular}{|c|c|c|c|c|c|c|c|c|c|c|c|c|c|c|c|}
\hline \multirow{2}{*}{ Samples } & \multicolumn{4}{|c|}{ Beidao Granite (TDG) } & \multicolumn{3}{|c|}{ Caochuanpu Granite (TCG) } & \multicolumn{6}{|c|}{ Yuanlongzhen Granite (TYG) } & \multicolumn{2}{|c|}{ Roche Type Rock (RTR) } \\
\hline & TDG-1 & TDG-2 & TDG-3 & TDG-4 & TCG-1 & TCG-2 & TCG-3 & TYG-1 & TYG-2 & TYG-3 & TYG-4 & TYG-5 & TYG-6 & RTR-1 & RTR-2 \\
\hline $\mathrm{SiO}_{2}$ (wt. \%) & 72.35 & 71.99 & 72.02 & 72.61 & 73.73 & 72.99 & 72.33 & 73.54 & 73.47 & 72.98 & 74.02 & 73.99 & 74.05 & 71.09 & 70.97 \\
\hline $\mathrm{Al}_{2} \mathrm{O}_{3}$ & 11.45 & 12.01 & 11.9 & 12.16 & 12.11 & 12.18 & 11.99 & 13.08 & 13.4 & 12.99 & 13.09 & 13.06 & 12.99 & 11.07 & 12.1 \\
\hline $\mathrm{MgO}$ & 0.32 & 0.38 & 0.48 & 1.66 & 0.14 & 0.29 & 0.36 & 0.14 & 0.21 & 0.23 & 0.46 & 0.38 & 0.34 & 0.98 & 0.83 \\
\hline $\mathrm{Fe}_{2} \mathrm{O}_{3}$ & 0.41 & 0.39 & 0.46 & 0.78 & 1.16 & 1.23 & 1.89 & 1.1 & 1.5 & 1.59 & 1.35 & 1.28 & 1.43 & 6.86 & 5.9 \\
\hline $\mathrm{CaO}$ & 0.37 & 0.45 & 0.32 & 1.58 & 0.76 & 0.89 & 0.9 & 0.88 & 0.99 & 0.86 & 0.96 & 1.06 & 1.09 & 0.45 & 0.58 \\
\hline $\mathrm{Na}_{2} \mathrm{O}$ & 3.33 & 3.43 & 3.35 & 3.38 & 3.7 & 3.9 & 3.7 & 3.64 & 3.4 & 3.45 & 3.3 & 3.42 & 3.5 & 1.13 & 1.59 \\
\hline $\mathrm{K}_{2} \mathrm{O}$ & 5.78 & 5.5 & 5.8 & 5.94 & 5.27 & 4.99 & 4.97 & 5.5 & 5.8 & 4.99 & 5.69 & 5.8 & 4.9 & 0.5 & 0.84 \\
\hline $\mathrm{CO}_{3}$ & 5.61 & 4.99 & 5.01 & 1.44 & 2.7 & 2.9 & 3.02 & 1.65 & 1.2 & 2.08 & 0.81 & 0.89 & 0.93 & 7.2 & 6.6 \\
\hline Total & 99.62 & 99.14 & 99.34 & 99.55 & 99.57 & 99.37 & 99.16 & 99.53 & 99.97 & 99.17 & 99.68 & 99.88 & 99.23 & 99.28 & 99.41 \\
\hline Mg\# & 65 & 69 & 70 & 83 & 21 & 35 & 30 & 22 & 24 & 25 & 44 & 40 & 35 & 24 & 24 \\
\hline \multicolumn{16}{|c|}{ Trace and rare-earth-elements (in ppm) } \\
\hline $\mathrm{Li}$ & 6.73 & 5.78 & 6.25 & 7.40 & 21.03 & 23.70 & 22.36 & 6.41 & 7.28 & 6.8 & 26.38 & 30.11 & 28.25 & 3.15 & 2.94 \\
\hline $\mathrm{Be}$ & 1.13 & 0.93 & 1.03 & 0.62 & 1.41 & 1.86 & 1.64 & 4.20 & 5.25 & 4.73 & 7.93 & 10.18 & 9.06 & 3.67 & 2.76 \\
\hline Sc & 1.78 & 1.54 & 1.65 & 3.14 & 1.91 & 2.22 & 2.06 & 3.29 & 3.82 & 3.56 & 2.72 & 3.10 & 2.91 & 8.63 & 7.63 \\
\hline
\end{tabular}


Table 2. Cont

\begin{tabular}{|c|c|c|c|c|c|c|c|c|c|c|c|c|c|c|c|}
\hline \multirow{2}{*}{ Samples } & \multicolumn{4}{|c|}{ Beidao Granite (TDG) } & \multicolumn{3}{|c|}{ Caochuanpu Granite (TCG) } & \multicolumn{6}{|c|}{ Yuanlongzhen Granite (TYG) } & \multicolumn{2}{|c|}{ Roche Type Rock (RTR) } \\
\hline & TDG-1 & TDG-2 & TDG-3 & TDG-4 & TCG-1 & TCG-2 & TCG-3 & TYG-1 & TYG-2 & TYG-3 & TYG-4 & TYG-5 & TYG-6 & RTR-1 & RTR-2 \\
\hline $\mathrm{V}$ & 11.65 & 14.04 & 12.84 & 18.50 & 23.62 & 19.65 & 21.63 & 30.07 & 24.92 & 27.49 & 49.56 & 41.13 & 45.34 & 16.10 & 19.32 \\
\hline $\mathrm{Cr}$ & 15.63 & 16.58 & 16.1 & 10.49 & 36.61 & 34.70 & 35.65 & 12.56 & 11.83 & 12.2 & 12.44 & 11.76 & 12.10 & 18.75 & 20.12 \\
\hline $\mathrm{Ni}$ & 7.24 & 7.34 & 7.29 & 7.47 & 6.32 & 6.43 & 6.37 & 3.33 & 3.31 & 3.32 & 5.82 & 5.81 & 5.81 & 3.96 & 3.95 \\
\hline $\mathrm{Cu}$ & 3.17 & 3.43 & 3.29 & 3.14 & 4.44 & 4.07 & 4.25 & 3.47 & 3.26 & 3.363 & 3.44 & 3.13 & 3.28 & 1.77 & 1.91 \\
\hline $\mathrm{Zn}$ & 16.73 & 21.02 & 18.87 & 22.26 & 21.08 & 17.37 & 19.22 & 25.48 & 20.28 & 22.88 & 42.15 & 33.62 & 37.89 & 203.13 & 255.50 \\
\hline $\mathrm{Ga}$ & 11.12 & 12.70 & 11.9 & 14.69 & 11.31 & 10.00 & 10.65 & 17.70 & 15.60 & 16.64 & 19.45 & 17.16 & 18.30 & 47.85 & 54.63 \\
\hline $\mathrm{Rb}$ & 127 & 148 & 137 & 262 & 113 & 99 & 106 & 206 & 175 & 191 & 293 & 252 & 273 & 16 & 18 \\
\hline $\mathrm{Sr}$ & 193 & 199 & 196 & 73 & 9 & 8 & 8 & 125 & 121 & 123 & 105 & 101 & 103 & 5 & 5 \\
\hline Y & 3.61 & 3.11 & 3.36 & 12.75 & 4.32 & 5.09 & 4.7 & 10.35 & 12.05 & 19.2 & 14.07 & 15.10 & 14.59 & 2.37 & 2.32 \\
\hline $\mathrm{Zr}$ & 260.39 & 267.48 & 263.93 & 123.03 & 27.54 & 27.36 & 27.45 & 115.78 & 112.73 & 114.25 & 161.16 & 157.29 & 159.23 & 4.59 & 4.77 \\
\hline $\mathrm{Ti}$ & 369 & 350 & 380 & 896 & 585 & 530 & 600 & 1019 & 1030 & 1000 & 773 & 756 & 764 & 2685 & 2601 \\
\hline $\mathrm{Nb}$ & 1.36 & 1.34 & 1.34 & 10.08 & 3.25 & 3.30 & 3.27 & 20.18 & 20.51 & 20.34 & 32.92 & 33.54 & 33.23 & 1.11 & 1.10 \\
\hline $\mathrm{Rh}$ & 71,336 & 71,478 & 71,407 & 67,537 & 69,045 & 68,874 & 68,959 & 71,816 & 72,008 & 71,912 & 68,844 & 68,887 & 68,866 & 73,188 & 72,995 \\
\hline Cs & 2.98 & 3.16 & 3.06 & 3.18 & 9.70 & 9.20 & 9.45 & 11.64 & 10.97 & 11.3 & 9.96 & 9.40 & 9.68 & 3.28 & 3.47 \\
\hline $\mathrm{Ba}$ & 1017 & 1145 & 1081 & 164 & 299 & 268 & 284 & 615 & 547 & 581 & 502 & 444 & 473 & 8 & 9 \\
\hline $\mathrm{La}$ & 26.27 & 27.32 & 26.79 & 64.46 & 9.39 & 9.08 & 9.23 & 26.12 & 25.02 & 25.56 & 45.91 & 44.00 & 44.95 & 1.91 & 1.99 \\
\hline $\mathrm{Ce}$ & 44.40 & 47.77 & 46.084 & 132.71 & 17.60 & 16.43 & 17.01 & 53.57 & 49.60 & 51.58 & 88.44 & 81.83 & 85.14 & 5.36 & 5.81 \\
\hline $\operatorname{Pr}$ & 4.35 & 4.83 & 4.59 & 14.60 & 1.79 & 1.63 & 1.71 & 5.06 & 4.55 & 4.8 & 10.08 & 9.05 & 9.57 & 0.31 & 0.34 \\
\hline $\mathrm{Nd}$ & 15.03 & 15.84 & 15.43 & 52.45 & 5.87 & 5.65 & 5.76 & 17.19 & 16.26 & 16.72 & 35.64 & 33.78 & 34.71 & 0.99 & 1.06 \\
\hline Sm & 2.49 & 2.53 & 2.51 & 11.40 & 1.11 & 1.12 & 1.11 & 3.24 & 3.18 & 3.21 & 6.91 & 6.83 & 6.87 & 0.20 & 0.20 \\
\hline $\mathrm{Eu}$ & 0.80 & 0.87 & 0.83 & 0.53 & 0.17 & 0.16 & 0.17 & 0.49 & 0.45 & 0.47 & 0.60 & 0.55 & 0.58 & 0.02 & 0.03 \\
\hline $\mathrm{Gd}$ & 1.66 & 2.41 & 2.03 & 7.17 & 1.19 & 0.85 & 1.02 & 3.13 & 2.17 & 2.64 & 6.39 & 4.45 & 5.42 & 0.15 & 0.21 \\
\hline $\mathrm{Tb}$ & 0.17 & 0.26 & 0.21 & 0.78 & 0.18 & 0.12 & 0.15 & 0.46 & 0.31 & 0.38 & 0.94 & 0.63 & 0.79 & 0.02 & 0.02 \\
\hline Dy & 0.84 & 1.10 & 0.97 & 3.25 & 0.97 & 0.78 & 0.87 & 2.58 & 2.00 & 2.28 & 4.85 & 3.84 & 4.34 & 0.08 & 0.10 \\
\hline Ho & 0.14 & 0.14 & 0.13 & 0.49 & 0.16 & 0.15 & 0.15 & 0.46 & 0.44 & 0.44 & 0.85 & 0.80 & 0.83 & 0.01 & 0.01 \\
\hline Er & 0.28 & 0.40 & 0.34 & 0.85 & 0.44 & 0.32 & 0.38 & 1.43 & 1.02 & 1.22 & 2.55 & 1.85 & 2.20 & 0.03 & 0.04 \\
\hline $\mathrm{Tm}$ & 0.04 & 0.04 & 0.041 & 0.08 & 0.05 & 0.05 & 0.05 & 0.20 & 0.18 & 0.18 & 0.37 & 0.33 & 0.35 & 0.01 & 0.01 \\
\hline $\mathrm{Yb}$ & 0.27 & 0.31 & 0.29 & 0.48 & 0.36 & 0.32 & 0.34 & 1.49 & 1.31 & 1.4 & 2.51 & 2.20 & 2.36 & 0.04 & 0.04 \\
\hline $\mathrm{Lu}$ & 0.04 & 0.05 & 0.046 & 0.07 & 0.05 & 0.04 & 0.043 & 0.21 & 0.19 & 0.2 & 0.36 & 0.32 & 0.34 & 0.01 & 0.01 \\
\hline $\mathrm{Hf}$ & 11.79 & 14.18 & 12.98 & 7.71 & 6.73 & 5.64 & 6.18 & 10.29 & 8.52 & 9.4 & 9.99 & 8.26 & 9.13 & 2.73 & 3.33 \\
\hline $\mathrm{Ta}$ & 0.12 & 0.11 & 0.11 & 0.47 & 0.31 & 0.33 & 0.32 & 1.80 & 1.93 & 1.86 & 2.06 & 2.20 & 2.13 & 0.34 & 0.32 \\
\hline $\mathrm{Pb}$ & 28.98 & 32.98 & 30.97 & 51.31 & 8.07 & 7.16 & 7.6 & 29.68 & 26.12 & 27.89 & 38.85 & 34.24 & 36.55 & 4.26 & 4.85 \\
\hline Th & 18.35 & 16.18 & 17.26 & 70.88 & 5.41 & 6.21 & 5.81 & 12.11 & 13.69 & 12.89 & 33.14 & 37.72 & 35.43 & 1.14 & 1.01 \\
\hline $\mathrm{U}$ & 1.28 & 1.25 & 1.26 & 2.85 & 0.98 & 1.01 & 0.99 & 3.60 & 3.68 & 3.63 & 5.65 & 5.80 & 5.73 & 0.22 & 0.22 \\
\hline
\end{tabular}


Table 2. Cont

\begin{tabular}{|c|c|c|c|c|c|c|c|c|c|c|c|c|c|c|c|}
\hline \multirow{2}{*}{ Samples } & \multicolumn{4}{|c|}{ Beidao Granite (TDG) } & \multicolumn{3}{|c|}{ Caochuanpu Granite (TCG) } & \multicolumn{6}{|c|}{ Yuanlongzhen Granite (TYG) } & \multicolumn{2}{|c|}{ Roche Type Rock (RTR) } \\
\hline & TDG-1 & TDG-2 & TDG-3 & TDG-4 & TCG-1 & TCG-2 & TCG-3 & TYG-1 & TYG-2 & TYG-3 & TYG-4 & TYG-5 & TYG-6 & RTR-1 & RTR-2 \\
\hline $\mathrm{Rb} / \mathrm{Sr}$ & 0.65 & 0.74 & 0.70 & 3.59 & 12.63 & 11.30 & 11.98 & 1.63 & 1.45 & 1.54 & 2.78 & 2.47 & 2.63 & 3.21 & 3.57 \\
\hline $\mathrm{Eu} / \mathrm{Eu}^{*}$ & 1.20 & 1.07 & 1.12 & 0.18 & 0.46 & 0.50 & 0.49 & 0.46 & 0.52 & 0.49 & 0.27 & 0.30 & 0.29 & 0.40 & 0.38 \\
\hline $\mathrm{Ce} / \mathrm{Ce}^{*}$ & 0.99 & 1.00 & 1.00 & 1.04 & 1.03 & 1.02 & 1.03 & 1.12 & 1.11 & 1.12 & 0.99 & 0.98 & 0.98 & 1.67 & 1.69 \\
\hline$(\mathrm{La} / \mathrm{Yb})_{\mathrm{N}}$ & 67.26 & 60.55 & 64.00 & 93.43 & 17.97 & 19.68 & 18.81 & 12.13 & 13.21 & 12.65 & 12.67 & 13.85 & 13.22 & 34.80 & 31.35 \\
\hline$\sum R E E$ & 96.79 & 103.88 & 100.29 & 289.33 & 39.34 & 36.71 & 37.99 & 115.62 & 106.67 & 111.08 & 206.40 & 190.46 & 198.43 & 9.12 & 9.86 \\
\hline $\bar{\Sigma} \mathrm{LREE}$ & 92.54 & 98.30 & 95.40 & 275.63 & 35.77 & 33.91 & 34.82 & 105.18 & 98.60 & 101.87 & 186.98 & 175.49 & 181.24 & 8.76 & 9.40 \\
\hline$\sum$ HREE & 4.25 & 5.58 & 4.89 & 13.70 & 3.58 & 2.80 & 3.17 & 10.44 & 8.07 & 9.21 & 19.42 & 14.97 & 17.19 & 0.35 & 0.46 \\
\hline$\sum \mathrm{LREE} / \sum \mathrm{HREE}$ & 21.77 & 17.60 & 19.52 & 20.11 & 10.00 & 12.13 & 10.97 & 10.070 & 12.22 & 11.06 & 9.63 & 11.72 & 10.54 & 24.95 & 20.29 \\
\hline
\end{tabular}

$\mathrm{Eu} / \mathrm{Eu}^{*}=\mathrm{Eu}_{N} /\left(\mathrm{Sm}_{\mathrm{N}} \times \mathrm{Gd}_{\mathrm{N}}\right)^{1 / 2}$ and $\mathrm{Ce} / \mathrm{Ce}^{*}=\mathrm{Ce}_{\mathrm{N}} /\left(\mathrm{La}_{\mathrm{N}} \times \mathrm{Pr}_{\mathrm{N}}\right)^{1 / 2}$

Table 3. Zircon LA-ICP-MS U-Pb results for the Tianshui area granitoids.

\begin{tabular}{|c|c|c|c|c|c|c|c|c|c|c|c|c|c|c|c|c|}
\hline \multirow{2}{*}{$\begin{array}{l}\text { Contents } \\
\text { (ESIS) }\end{array}$} & \multirow{2}{*}{$\begin{array}{c}\text { Th } \\
\text { ppm }\end{array}$} & \multirow{2}{*}{$\frac{\mathrm{U}}{\mathrm{ppm}}$} & \multirow{2}{*}{$\begin{array}{l}\mathrm{Th} / \mathrm{U} \\
\text { Ratio }\end{array}$} & \multicolumn{2}{|c|}{${ }^{207} \mathrm{~Pb} /{ }^{206} \mathrm{~Pb}$} & \multicolumn{2}{|c|}{${ }^{207} \mathrm{~Pb} /{ }^{235} \mathrm{U}$} & \multicolumn{2}{|c|}{${ }^{206} \mathrm{~Pb} /{ }^{238} \mathrm{U}$} & \multicolumn{2}{|c|}{${ }^{207} \mathrm{~Pb} /{ }^{206} \mathrm{~Pb}$} & \multicolumn{2}{|c|}{${ }^{207} \mathrm{~Pb} /{ }^{235} \mathrm{U}$} & \multicolumn{2}{|c|}{${ }^{206} \mathrm{~Pb} /{ }^{238} \mathrm{U}$} & \multirow[b]{2}{*}{ Concordance } \\
\hline & & & & Ratio & $1 \sigma$ & Ratio & $1 \sigma$ & Ratio & $1 \sigma$ & Age (Ma) & $1 \sigma$ & Age (Ma) & $1 \sigma$ & Age (Ma) & $1 \sigma$ & \\
\hline \multicolumn{17}{|c|}{ Beidao granite (TDG) } \\
\hline TDG-1-1 & 86 & 738 & 0.117 & 0.054 & 0.001 & 0.500 & 0.014 & 0.067 & 0.002 & 390 & 25 & 411 & 9 & 415 & 11 & $99 \%$ \\
\hline TDG-1-2 & 168 & 791 & 0.213 & 0.055 & 0.001 & 0.506 & 0.015 & 0.067 & 0.002 & 405 & 21 & 415 & 9 & 417 & 11 & $99 \%$ \\
\hline TDG-1-3 & 68 & 661 & 0.103 & 0.057 & 0.001 & 0.522 & 0.013 & 0.067 & 0.002 & 476 & 25 & 426 & 9 & 417 & 11 & $97 \%$ \\
\hline TDG-1-4 & 67 & 622 & 0.108 & 0.056 & 0.001 & 0.509 & 0.012 & 0.066 & 0.002 & 453 & 25 & 417 & 8 & 411 & 10 & $98 \%$ \\
\hline TDG-1-5 & 95 & 715 & 0.133 & 0.056 & 0.001 & 0.509 & 0.012 & 0.066 & 0.002 & 455 & 25 & 418 & 8 & 412 & 9 & $98 \%$ \\
\hline TDG-1-6 & 67 & 626 & 0.107 & 0.056 & 0.001 & 0.512 & 0.016 & 0.066 & 0.002 & 450 & 31 & 419 & 10 & 412 & 11 & $98 \%$ \\
\hline TDG-1-7 & 131 & 675 & 0.195 & 0.056 & 0.001 & 0.517 & 0.012 & 0.067 & 0.001 & 435 & 24 & 423 & 8 & 419 & 9 & $99 \%$ \\
\hline TDG-1-8 & 113 & 601 & 0.187 & 0.060 & 0.001 & 0.560 & 0.027 & 0.068 & 0.004 & 613 & 33 & 451 & 17 & 422 & 22 & $93 \%$ \\
\hline TDG-1-9 & 47 & 540 & 0.087 & 0.056 & 0.001 & 0.523 & 0.015 & 0.067 & 0.002 & 453 & 26 & 426 & 10 & 419 & 10 & $98 \%$ \\
\hline TDG-1-10 & 109 & 606 & 0.179 & 0.070 & 0.002 & 0.660 & 0.029 & 0.068 & 0.002 & 920 & 50 & 514 & 17 & 423 & 13 & $80 \%$ \\
\hline TDG-1-11 & 98 & 740 & 0.132 & 0.058 & 0.001 & 0.535 & 0.013 & 0.067 & 0.001 & 600 & 27 & 435 & 8 & 415 & 9 & $95 \%$ \\
\hline TDG-1-12 & 98 & 1043 & 0.094 & 0.057 & 0.001 & 0.529 & 0.011 & 0.067 & 0.001 & 498 & 23 & 431 & 7 & 417 & 8 & $96 \%$ \\
\hline TDG-1-13 & 57 & 500 & 0.114 & 0.058 & 0.001 & 0.536 & 0.018 & 0.067 & 0.002 & 546 & 40 & 435 & 11 & 415 & 12 & $95 \%$ \\
\hline TDG-1-14 & 77 & 631 & 0.122 & 0.054 & 0.001 & 0.500 & 0.016 & 0.067 & 0.002 & 390 & 39 & 412 & 10 & 418 & 14 & $98 \%$ \\
\hline TDG-1-15 & 66 & 670 & 0.098 & 0.057 & 0.001 & 0.526 & 0.016 & 0.067 & 0.002 & 476 & 27 & 429 & 10 & 420 & 13 & $98 \%$ \\
\hline TDG-1-16 & 114 & 723 & 0.157 & 0.057 & 0.001 & 0.526 & 0.016 & 0.067 & 0.002 & 494 & 33 & 429 & 10 & 418 & 13 & $97 \%$ \\
\hline TDG-1-17 & 61 & 569 & 0.107 & 0.058 & 0.002 & 0.523 & 0.012 & 0.067 & 0.002 & 522 & 60 & 427 & 7 & 417 & 11 & $97 \%$ \\
\hline TDG-1-18 & 72 & 522 & 0.139 & 0.055 & 0.001 & 0.515 & 0.016 & 0.067 & 0.002 & 431 & 43 & 421 & 10 & 419 & 12 & $99 \%$ \\
\hline TDG-1-19 & 132 & 593 & 0.222 & 0.056 & 0.001 & 0.527 & 0.016 & 0.068 & 0.002 & 464 & 32 & 429 & 10 & 422 & 12 & $98 \%$ \\
\hline
\end{tabular}


Table 3. Cont

\begin{tabular}{|c|c|c|c|c|c|c|c|c|c|c|c|c|c|c|c|c|}
\hline \multirow{2}{*}{$\begin{array}{l}\text { Contents } \\
\text { (ESIS) }\end{array}$} & \multirow{2}{*}{$\begin{array}{c}\text { Th } \\
\text { ppm }\end{array}$} & \multirow{2}{*}{$\begin{array}{c}\mathrm{U} \\
\mathrm{ppm}\end{array}$} & \multirow{2}{*}{$\begin{array}{l}\mathrm{Th} / \mathrm{U} \\
\text { Ratio }\end{array}$} & \multicolumn{2}{|c|}{${ }^{207} \mathrm{~Pb} /{ }^{206} \mathrm{~Pb}$} & \multicolumn{2}{|c|}{${ }^{207} \mathrm{~Pb} /{ }^{235} \mathrm{U}$} & \multicolumn{2}{|c|}{${ }^{206} \mathrm{~Pb} /{ }^{238} \mathrm{U}$} & \multicolumn{2}{|c|}{${ }^{207} \mathrm{~Pb} /{ }^{206} \mathrm{~Pb}$} & \multicolumn{2}{|c|}{${ }^{207} \mathrm{~Pb} /{ }^{235} \mathrm{U}$} & \multicolumn{2}{|c|}{${ }^{206} \mathrm{~Pb} /{ }^{238} \mathrm{U}$} & \multirow[b]{2}{*}{ Concordance } \\
\hline & & & & Ratio & $1 \sigma$ & Ratio & $1 \sigma$ & Ratio & $1 \sigma$ & Age (Ma) & $1 \sigma$ & Age (Ma) & $1 \sigma$ & Age (Ma) & $1 \sigma$ & \\
\hline \multicolumn{17}{|c|}{ Caochuanpu granite (TCG) } \\
\hline TCG-2-1 & 455 & 1147 & 0.396 & 0.051 & 0.001 & 0.23 & 0.008 & 0.033 & 0.001 & 227 & 35 & 211 & 6 & 210 & 8 & $99 \%$ \\
\hline TCG-2-2 & 309 & 440 & 0.703 & 0.062 & 0.004 & 0.29 & 0.020 & 0.034 & 0.001 & 664 & 125 & 258 & 15 & 214 & 4 & $81 \%$ \\
\hline TCG-2-3 & 158 & 294 & 0.538 & 0.053 & 0.002 & 0.25 & 0.010 & 0.034 & 0.001 & 331 & 68 & 223 & 8 & 213 & 5 & $95 \%$ \\
\hline TCG-2-4 & 461 & 873 & 0.527 & 0.050 & 0.001 & 0.24 & 0.008 & 0.034 & 0.001 & 187 & 65 & 214 & 6 & 217 & 4 & $98 \%$ \\
\hline TCG-2-5 & 103 & 193 & 0.532 & 0.052 & 0.002 & 0.24 & 0.010 & 0.034 & 0.001 & 283 & 83 & 221 & 8 & 216 & 5 & $97 \%$ \\
\hline TCG-2-6 & 679 & 1017 & 0.667 & 0.052 & 0.001 & 0.24 & 0.008 & 0.034 & 0.001 & 298 & 43 & 221 & 6 & 215 & 7 & $96 \%$ \\
\hline TCG-2-7 & 664 & 1055 & 0.629 & 0.060 & 0.002 & 0.29 & 0.008 & 0.035 & 0.001 & 613 & 74 & 259 & 6 & 222 & 4 & $84 \%$ \\
\hline TCG-2-8 & 235 & 375 & 0.626 & 0.051 & 0.001 & 0.24 & 0.008 & 0.034 & 0.001 & 242 & 59 & 218 & 6 & 215 & 5 & $98 \%$ \\
\hline TCG-2-9 & 198 & 409 & 0.484 & 0.058 & 0.003 & 0.26 & 0.008 & 0.034 & 0.001 & 542 & 103 & 236 & 6 & 213 & 7 & $89 \%$ \\
\hline TCG-2-10 & 209 & 377 & 0.554 & 0.055 & 0.003 & 0.25 & 0.009 & 0.034 & 0.001 & 466 & 120 & 225 & 7 & 214 & 6 & $95 \%$ \\
\hline TCG-2-11 & 633 & 1333 & 0.475 & 0.055 & 0.001 & 0.26 & 0.007 & 0.034 & 0.001 & 416 & 46 & 232 & 6 & 217 & 7 & $93 \%$ \\
\hline TCG-2-12 & 688 & 884 & 0.778 & 0.051 & 0.001 & 0.24 & 0.008 & 0.034 & 0.001 & 242 & 34 & 216 & 6 & 214 & 5 & $98 \%$ \\
\hline TCG-2-13 & 81 & 154 & 0.396 & 0.051 & 0.002 & 0.24 & 0.012 & 0.034 & 0.001 & 220 & 85 & 215 & 9 & 214 & 6 & $99 \%$ \\
\hline \multicolumn{17}{|c|}{ Yuanlongzhen granite (TYG) } \\
\hline TYG-1-1 & 122 & 234 & 0.525 & 0.064 & 0.002 & 0.244 & 0.008 & 0.036 & 0.010 & 242 & 52 & 219 & 6 & 218 & 6 & $86 \%$ \\
\hline TYG-1-2 & 211 & 339 & 0.521 & 0.050 & 0.001 & 0.241 & 0.007 & 0.035 & 0.001 & 205 & 53 & 218 & 6 & 220 & 4 & $99 \%$ \\
\hline TYG-1-3 & 88 & 136 & 0.622 & 0.051 & 0.002 & 0.240 & 0.012 & 0.034 & 0.001 & 253 & 80 & 218 & 9 & 216 & 5 & $98 \%$ \\
\hline TYG-1-4 & 160 & 250 & 0.652 & 0.050 & 0.002 & 0.244 & 0.009 & 0.035 & 0.001 & 216 & 49 & 221 & 7 & 222 & 4 & $99 \%$ \\
\hline TYG-1-5 & 330 & 430 & 0.639 & 0.050 & 0.001 & 0.236 & 0.008 & 0.034 & 0.001 & 187 & 42 & 215 & 6 & 217 & 5 & $99 \%$ \\
\hline TYG-1-6 & 484 & 711 & 0.767 & 0.052 & 0.001 & 0.245 & 0.007 & 0.034 & 0.001 & 333 & 31 & 222 & 5 & 217 & 5 & $98 \%$ \\
\hline TYG-1-7 & 409 & 454 & 0.681 & 0.049 & 0.002 & 0.226 & 0.010 & 0.034 & 0.001 & 200 & 99 & 206 & 8 & 213 & 5 & $96 \%$ \\
\hline TYG-1-8 & 185 & 301 & 0.901 & 0.053 & 0.001 & 0.253 & 0.010 & 0.035 & 0.001 & 316 & 57 & 229 & 8 & 220 & 7 & $96 \%$ \\
\hline TYG-1-9 & 300 & 411 & 0.614 & 0.056 & 0.002 & 0.268 & 0.008 & 0.035 & 0.001 & 442 & 68 & 241 & 6 & 220 & 4 & $91 \%$ \\
\hline TYG-1-10 & 263 & 375 & 0.729 & 0.051 & 0.001 & 0.243 & 0.007 & 0.035 & 0.001 & 233 & 51 & 220 & 5 & 220 & 5 & $99 \%$ \\
\hline TYG-1-11 & 182 & 304 & 0.700 & 0.053 & 0.002 & 0.249 & 0.010 & 0.034 & 0.001 & 322 & 66 & 225 & 7 & 216 & 5 & $96 \%$ \\
\hline TYG-1-12 & 192 & 348 & 0.598 & 0.053 & 0.001 & 0.251 & 0.008 & 0.035 & 0.001 & 324 & 61 & 227 & 6 & 220 & 7 & $96 \%$ \\
\hline TYG-1-13 & 165 & 465 & 0.552 & 0.053 & 0.001 & 0.253 & 0.011 & 0.035 & 0.001 & 324 & 62 & 229 & 8 & 219 & 6 & $95 \%$ \\
\hline
\end{tabular}


Table 4. The REE and other elements results (in ppm) for the analyzed zircons from the Tianshui granitoids.

\begin{tabular}{|c|c|c|c|c|c|c|c|c|c|c|c|c|c|c|c|c|c|c|c|}
\hline $\begin{array}{c}\text { Zircon Trace } \\
\text { Elements }\end{array}$ & La & $\mathrm{Ce}$ & Pr & Nd & Sm & Eu & Gd & $\mathrm{Tb}$ & Dy & Ho & Er & $\mathrm{Tm}$ & $\mathbf{Y b}$ & Lu & $\mathrm{Pb}$ & $\sum$ REE & LREE & HREE & LREE/HREE \\
\hline & \multicolumn{19}{|c|}{ Beidao Granite (TDG) Zircon } \\
\hline TDG-1-1 & 0.0 & 2.3 & 0.5 & 1.2 & 2.7 & 0.5 & 12.8 & 6.2 & 80.3 & 29.0 & 128.8 & 31.4 & 293.8 & 47.6 & 51.6 & 637.4 & 6.8 & 630.6 & 0.0108 \\
\hline TDG-1-2 & 1.3 & 3.3 & 0.0 & 1.6 & 2.6 & 0.0 & 20.9 & 9.8 & 109.7 & 36.4 & 156.1 & 34.1 & 332.2 & 51.1 & 56.7 & 759.2 & 8.9 & 750.4 & 0.0118 \\
\hline TDG-1-3 & 0.1 & 1.7 & 0.0 & 0.0 & 2.5 & 0.0 & 14.5 & 5.3 & 64.4 & 27.1 & 114.5 & 27.4 & 270.6 & 41.0 & 45.6 & 569.3 & 4.4 & 564.9 & 0.0078 \\
\hline TDG-1-4 & 0.0 & 1.9 & 0.2 & 0.0 & 2.6 & 0.0 & 14.1 & 5.6 & 67.7 & 23.0 & 105.1 & 24.8 & 241.1 & 38.0 & 43.4 & 524.2 & 4.8 & 519.4 & 0.0091 \\
\hline TDG-1-5 & 0.9 & 2.0 & 0.4 & 0.4 & 0.8 & 0.3 & 12.1 & 6.2 & 82.8 & 28.6 & 120.9 & 28.8 & 275.6 & 43.5 & 51.1 & 603.1 & 4.4 & 598.7 & 0.0074 \\
\hline TDG-1-6 & 0.0 & 0.5 & 0.0 & 0.8 & 2.4 & 0.0 & 14.8 & 5.5 & 64.6 & 22.3 & 97.3 & 23.0 & 229.5 & 36.7 & 44.7 & 497.4 & 3.7 & 493.7 & 0.0074 \\
\hline TDG-1-7 & 1.1 & 3.6 & 0.0 & 0.4 & 3.1 & 0.2 & 17.4 & 7.3 & 91.8 & 31.9 & 130.6 & 29.0 & 278.5 & 43.8 & 49.6 & 638.8 & 8.2 & 630.6 & 0.0129 \\
\hline TDG-1-8 & 2.1 & 18.0 & 1.3 & 16.0 & 14.8 & 3.8 & 30.4 & 7.9 & 83.2 & 27.4 & 112.4 & 27.3 & 242.6 & 38.5 & 43.1 & 625.8 & 52.4 & 573.4 & 0.0913 \\
\hline TDG-1-9 & 0.8 & 1.3 & 0.0 & 0.6 & 1.3 & 0.6 & 13.7 & 5.6 & 66.2 & 21.8 & 97.2 & 22.9 & 215.1 & 36.5 & 39.0 & 483.8 & 4.1 & 479.7 & 0.0084 \\
\hline TDG-1-10 & 0.0 & 5.8 & 0.5 & 6.7 & 5.5 & 1.9 & 17.5 & 6.6 & 71.4 & 24.7 & 102.7 & 24.1 & 241.7 & 36.1 & 44.3 & 545.2 & 18.4 & 526.8 & 0.0349 \\
\hline TDG-1-11 & 0.0 & 2.9 & 0.2 & 1.2 & 3.8 & 0.0 & 15.9 & 5.7 & 66.5 & 24.5 & 96.2 & 23.2 & 220.2 & 35.3 & 54.8 & 495.6 & 8.1 & 487.5 & 0.0167 \\
\hline TDG-1-12 & 0.0 & 2.9 & 0.0 & 0.0 & 2.0 & 0.0 & 12.6 & 4.2 & 61.5 & 23.9 & 104.5 & 24.4 & 234.2 & 38.1 & 76.1 & 508.4 & 4.9 & 503.4 & 0.0097 \\
\hline TDG-1-13 & 0.0 & 0.2 & 0.2 & 0.9 & 2.1 & 0.2 & 12.0 & 4.0 & 49.4 & 16.5 & 71.6 & 17.3 & 169.0 & 24.7 & 34.3 & 368.1 & 3.5 & 364.7 & 0.0094 \\
\hline TDG-1-14 & 0.0 & 1.3 & 0.5 & 1.0 & 3.2 & 0.2 & 16.4 & 6.6 & 72.8 & 23.7 & 105.8 & 23.9 & 221.4 & 34.6 & 44.4 & 511.4 & 5.9 & 505.5 & 0.0117 \\
\hline TDG-1-15 & 0.0 & 1.1 & 0.3 & 0.0 & 2.1 & 0.2 & 15.2 & 5.5 & 62.1 & 21.9 & 88.0 & 20.7 & 204.4 & 31.4 & 46.9 & 452.9 & 3.5 & 449.4 & 0.0077 \\
\hline TDG-1-16 & 0.0 & 1.6 & 0.3 & 0.4 & 1.7 & 0.3 & 16.1 & 7.8 & 82.3 & 29.2 & 124.2 & 28.6 & 274.6 & 41.3 & 52.3 & 608.4 & 4.1 & 604.3 & 0.0067 \\
\hline TDG-1-17 & 0.0 & 0.6 & 0.0 & 0.0 & 2.1 & 0.0 & 12.2 & 5.2 & 54.9 & 18.4 & 78.0 & 18.3 & 181.0 & 29.2 & 40.5 & 400.0 & 2.6 & 397.4 & 0.0066 \\
\hline TDG-1-18 & 0.3 & 1.7 & 0.0 & 0.0 & 0.0 & 0.0 & 10.8 & 4.5 & 53.4 & 20.1 & 86.7 & 18.4 & 195.6 & 31.4 & 37.2 & 423.1 & 2.1 & 421.0 & 0.0049 \\
\hline \multirow[t]{2}{*}{ TDG-1-19 } & 0.2 & 7.3 & 0.0 & 5.4 & 3.4 & 0.3 & 18.3 & 6.5 & 69.6 & 23.9 & 96.6 & 21.9 & 203.6 & 32.2 & 43.9 & 489.2 & 16.4 & 472.8 & 0.0346 \\
\hline & \multicolumn{19}{|c|}{ Caochuanpu granite (TCG) Zircon } \\
\hline TCG-2-1 & 1.0 & 16.2 & 0.2 & 3.1 & 1.1 & 0.0 & 15.7 & 6.9 & 83.1 & 28.0 & 122.1 & 27.4 & 265.7 & 43.0 & 44.5 & 613.5 & 21.7 & 591.8 & 0.0366 \\
\hline TCG-2-2 & 27.7 & 106.4 & 11.0 & 43.2 & 16.5 & 2.6 & 41.0 & 12.7 & 121.3 & 39.3 & 153.8 & 34.3 & 327.9 & 51.4 & 18.5 & 989.0 & 204.7 & 784.2 & 0.2610 \\
\hline TCG-2-3 & 1.4 & 26.2 & 0.0 & 1.8 & 3.3 & 1.2 & 11.8 & 3.7 & 46.0 & 15.3 & 66.4 & 14.8 & 150.2 & 24.7 & 11.9 & 366.6 & 32.6 & 334.1 & 0.0974 \\
\hline TCG-2-4 & 0.6 & 57.3 & 0.0 & 7.1 & 7.5 & 0.0 & 32.5 & 10.7 & 121.1 & 40.1 & 185.1 & 41.6 & 412.3 & 66.0 & 36.4 & 981.8 & 72.4 & 909.3 & 0.0796 \\
\hline TCG-2-5 & 1.0 & 21.5 & 1.1 & 1.9 & 3.7 & 0.2 & 10.2 & 3.1 & 35.4 & 12.0 & 52.4 & 11.5 & 125.5 & 20.2 & 8.1 & 299.6 & 29.1 & 270.5 & 0.1077 \\
\hline TCG-2-6 & 0.0 & 42.8 & 0.8 & 6.2 & 10.8 & 0.5 & 29.0 & 10.7 & 116.7 & 38.7 & 161.5 & 35.6 & 353.1 & 51.1 & 42.3 & 857.6 & 60.6 & 797.0 & 0.0760 \\
\hline TCG-2-7 & 0.9 & 31.9 & 2.1 & 9.0 & 16.4 & 0.8 & 44.2 & 13.8 & 142.7 & 44.6 & 189.9 & 38.6 & 370.2 & 60.2 & 45.5 & 965.2 & 60.3 & 904.9 & 0.0666 \\
\hline TCG-2-8 & 0.0 & 29.2 & 0.0 & 3.8 & 4.4 & 0.5 & 21.0 & 5.6 & 59.0 & 20.6 & 91.6 & 20.3 & 202.7 & 31.8 & 15.5 & 490.4 & 37.5 & 453.0 & 0.0827 \\
\hline TCG-2-9 & 35.6 & 138.7 & 15.7 & 93.3 & 29.2 & 0.9 & 30.8 & 7.0 & 69.2 & 21.4 & 81.9 & 17.8 & 196.2 & 28.9 & 15.8 & 766.6 & 312.5 & 454.1 & 0.6882 \\
\hline
\end{tabular}


Table 4. Cont.

\begin{tabular}{|c|c|c|c|c|c|c|c|c|c|c|c|c|c|c|c|c|c|c|c|}
\hline $\begin{array}{l}\text { Zircon Trace } \\
\text { Elements }\end{array}$ & La & $\mathrm{Ce}$ & Pr & $\mathrm{Nd}$ & Sm & $\mathbf{E u}$ & Gd & $\mathbf{T b}$ & Dy & Ho & Er & $\mathrm{Tm}$ & $\mathrm{Yb}$ & $\mathbf{L u}$ & $\mathrm{Pb}$ & $\sum$ REE & LREE & HREE & LREE/HREE \\
\hline TCG-2-10 & 0.0 & 31.6 & 0.0 & 1.3 & 4.5 & 0.8 & 23.1 & 5.0 & 56.4 & 18.8 & 74.8 & 17.0 & 181.5 & 27.3 & 15.1 & 442.1 & 37.4 & 404.7 & 0.0923 \\
\hline TCG-2-11 & 13.7 & 56.9 & 5.2 & 30.9 & 7.8 & 0.5 & 38.3 & 11.7 & 133.3 & 43.0 & 181.7 & 40.7 & 395.9 & 56.6 & 52.1 & 1016.0 & 114.4 & 901.6 & 0.1269 \\
\hline TCG-2-12 & 0.5 & 55.1 & 0.0 & 6.1 & 3.0 & 1.3 & 36.8 & 10.8 & 124.6 & 46.1 & 188.0 & 43.0 & 444.6 & 66.3 & 37.1 & 1026.2 & 64.7 & 961.4 & 0.0673 \\
\hline \multirow[t]{2}{*}{ TCG-2-13 } & 0.0 & 13.1 & 0.0 & 1.1 & 2.9 & 2.0 & 8.1 & 3.2 & 33.9 & 11.5 & 49.5 & 11.8 & 118.0 & 18.7 & 6.2 & 273.8 & 17.1 & 256.7 & 0.0666 \\
\hline & \multicolumn{19}{|c|}{ Yuanlongzhen granite (TYG) Zircon } \\
\hline TYG-1-1 & 0.5 & 32.3 & 0.8 & 0.2 & 2.9 & 0.2 & 18.0 & 6.1 & 71.5 & 29.2 & 131.3 & 30.3 & 292.4 & 47.1 & 20.8 & 662.8 & 36.6 & 626.2 & 0.0584 \\
\hline TYG-1-2 & 0.3 & 48.5 & 0.0 & 4.7 & 2.7 & 0.6 & 17.8 & 6.4 & 76.3 & 29.8 & 135.5 & 31.4 & 308.4 & 51.3 & 14.3 & 713.6 & 56.1 & 657.5 & 0.0854 \\
\hline TYG-1-3 & 0.1 & 32.2 & 0.2 & 0.0 & 1.3 & 0.7 & 17.2 & 4.6 & 54.4 & 20.9 & 89.3 & 20.2 & 217.2 & 36.3 & 5.8 & 494.8 & 33.8 & 461.0 & 0.0733 \\
\hline TYG-1-4 & 1.4 & 41.0 & 0.0 & 3.4 & 2.5 & 0.3 & 18.4 & 5.5 & 71.1 & 28.3 & 135.0 & 29.6 & 304.2 & 52.0 & 11.0 & 693.0 & 48.3 & 644.7 & 0.0749 \\
\hline TYG-1-5 & 0.7 & 65.7 & 0.6 & 2.9 & 5.7 & 1.6 & 22.6 & 8.1 & 94.2 & 35.1 & 157.7 & 37.2 & 351.1 & 57.8 & 18.7 & 841.0 & 75.6 & 765.4 & 0.0987 \\
\hline TYG-1-6 & 2.2 & 75.8 & 0.9 & 3.9 & 7.9 & 1.5 & 33.6 & 11.9 & 137.3 & 50.6 & 220.2 & 50.1 & 515.8 & 85.8 & 31.1 & 1197.3 & 90.6 & 1106.7 & 0.0818 \\
\hline TYG-1-7 & 3.6 & 53.3 & 0.0 & 5.9 & 7.7 & 1.8 & 29.7 & 7.4 & 81.6 & 32.6 & 134.2 & 29.9 & 297.1 & 47.0 & 20.2 & 731.7 & 70.6 & 661.2 & 0.1067 \\
\hline TYG-1-8 & 0.8 & 51.0 & 0.0 & 0.0 & 0.5 & 1.2 & 15.7 & 5.9 & 69.7 & 25.9 & 109.0 & 25.6 & 264.3 & 41.9 & 12.3 & 611.5 & 52.3 & 559.2 & 0.0935 \\
\hline TYG-1-9 & 0.1 & 51.1 & 0.3 & 1.0 & 1.1 & 1.4 & 14.3 & 4.9 & 70.4 & 26.6 & 121.6 & 25.7 & 283.2 & 45.4 & 18.4 & 646.9 & 53.6 & 593.4 & 0.0902 \\
\hline TYG-1-10 & 2.2 & 48.5 & 1.2 & 4.4 & 4.5 & 0.7 & 21.8 & 6.4 & 67.6 & 24.9 & 106.7 & 24.8 & 252.8 & 39.9 & 16.5 & 606.5 & 60.8 & 545.7 & 0.1114 \\
\hline TYG-1-11 & 0.1 & 52.2 & 1.0 & 5.2 & 4.5 & 1.8 & 21.4 & 5.7 & 71.6 & 24.1 & 107.1 & 26.6 & 272.1 & 44.2 & 12.7 & 637.7 & 63.0 & 574.7 & 0.1095 \\
\hline TYG-1-12 & 1.3 & 51.5 & 0.3 & 2.5 & 6.3 & 2.2 & 27.5 & 8.9 & 103.9 & 38.2 & 163.6 & 41.4 & 436.5 & 68.8 & 14.1 & 952.9 & 62.0 & 890.9 & 0.0695 \\
\hline TYG-1-13 & 2.3 & 12.6 & 0.0 & 2.2 & 0.7 & 1.1 & 16.3 & 7.0 & 74.5 & 29.1 & 130.1 & 33.0 & 344.4 & 55.4 & 18.1 & 708.7 & 17.8 & 690.9 & 0.0257 \\
\hline
\end{tabular}




\section{Results}

Three main types of granitoids (i.e., Beidao granite, Yuanlongzhen granite, Caochuanpu granite) and the Roche type rocks were investigated in this study. Below we provide their details.

\subsection{Petrography}

\subsubsection{Beidao and Caochuanpu Granites}

The Beidao granite intrudes the Paleoproterozoic and Early Paleozoic metamorphic rocks of the Qinling Group; the granite cuts the regional metamorphic schistosity (Figure 1b). White to pink color indicates fresh whereas yellow color shows weathered granite (Figure 2a,b). Texturally, the Beidao granite is medium- to coarse-grained with anhedral to subhedral grains exhibiting a hypidiomorphic texture (Figure 3a). Mineralogically, it is composed of quartz (27-35 mode \%), plagioclase (24-30\%), microcline/microcline perthite (25-33\%), biotite (1-3\%), muscovite (up to 1\%), with accessory minerals (zircon: $0.5-3 \%$, fluorapatite: $0.1 \%$, rutile: $0.1 \%$ ), secondary sericite (3-9\%), and opaque minerals (1-2\%) (Table 1). Quartz crystals are anhedral to subhedral and most of the grains show undulose extinction (Figure 3b). The alkali feldspar (microcline and microcline perthite) is the second most abundant mineral in the granite. In the microcline perthite, exsolution lamellae with irregular bleb thicknesses are observed (Figure 3b). Quartz and feldspars grains are highly fractured, suggesting local tectonic activities that possibly occurred along faults. In most samples, myrmekitic texture is common suggesting temperature fluctuations during feldspar and quartz crystallization (Figure 3b). Muscovite flakes within and around biotite suggest their formation at the expense of biotite alteration (Figure 3c). Biotite flakes contain abundant zircon inclusions indicating its early crystallization and that of zircon. Zircon crystals range from 50-350 $\mu \mathrm{m}$, with some exceptionally larger grains reaching up to $450 \mu \mathrm{m}$. Zircon crystals also contain melt inclusions (Figure 3d).

(a)

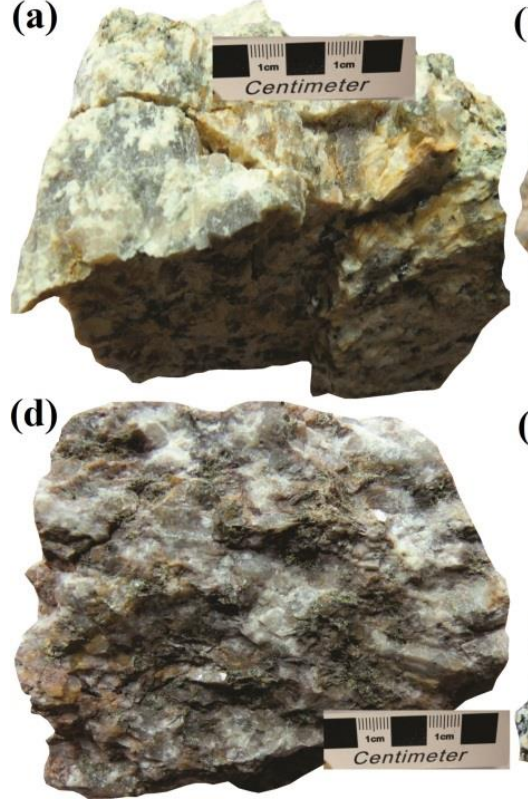

(b)

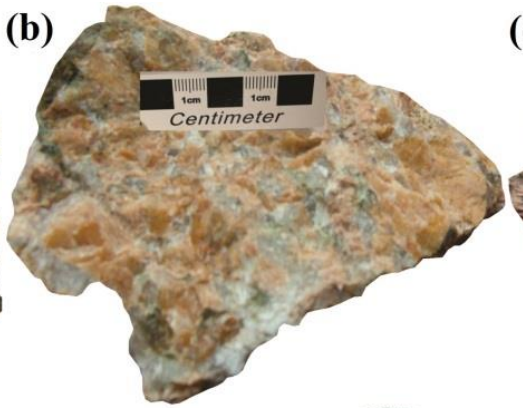

(e)

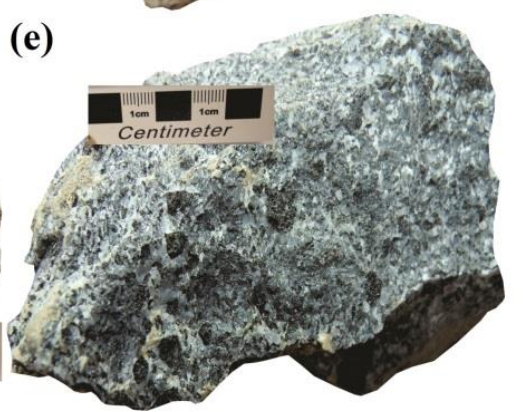

(c)

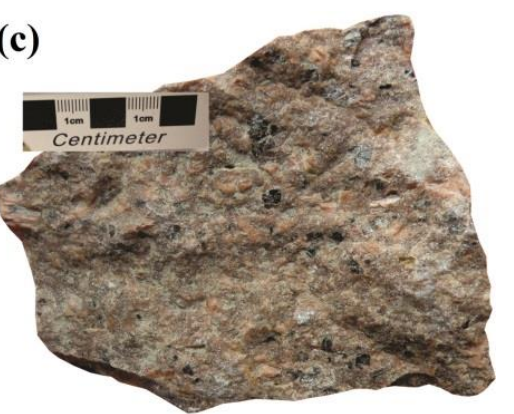

Figure 2. Photographs of the granitoid samples used for this study (a) fresh and weathered color of the Beidao granite (TDG-1), (b) orange variety of the Beidao granite (TDG-4), (c) porphyritic texture of the Caochuanpu granite (TCG-2), (d) pink variety of very coarse-grained Yuanlongzhen granite (TYG-1), and (e) Roche type rock (RTR-1) containing quartz and tourmaline crystals. 

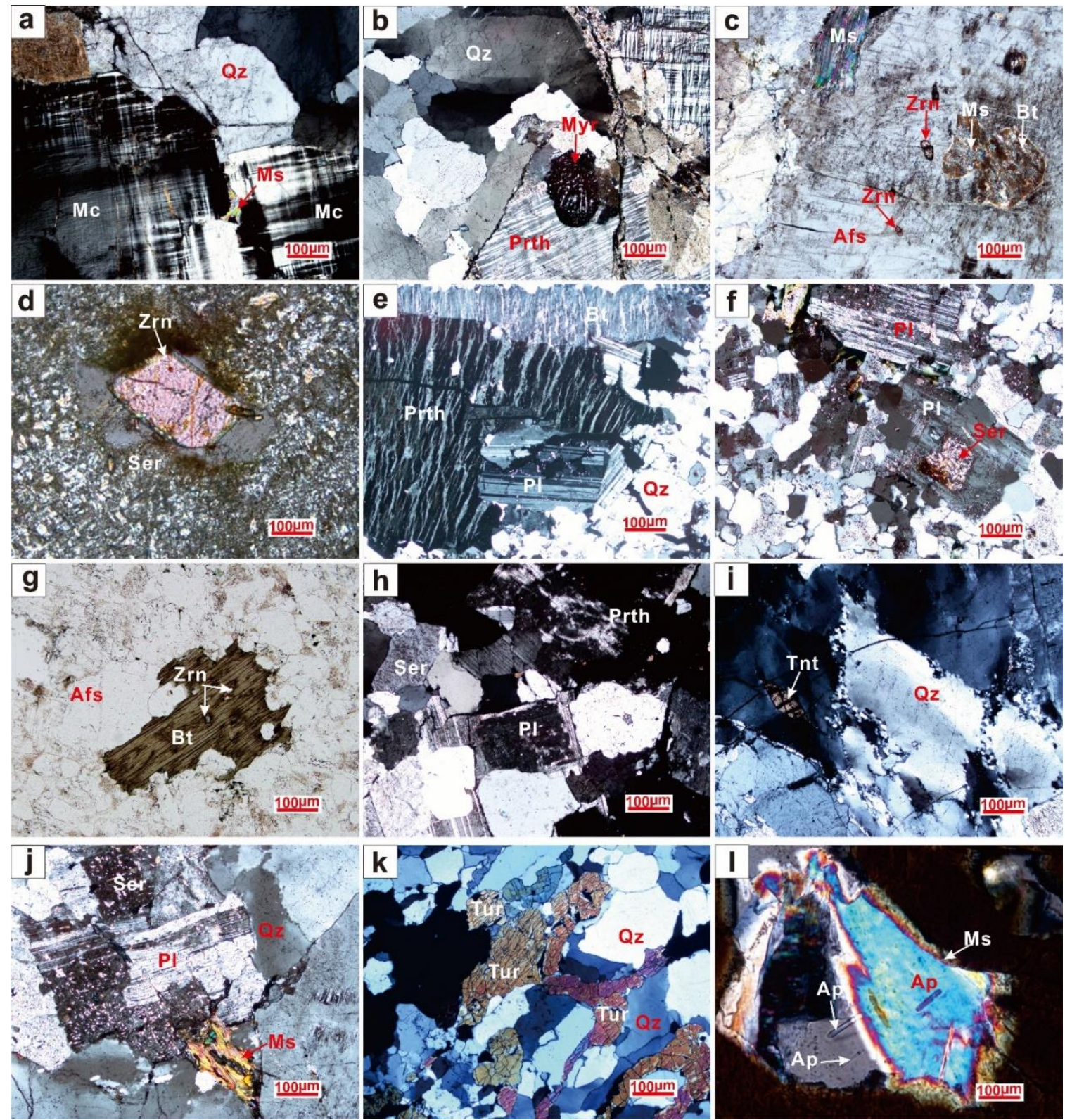

Figure 3. Photomicrographs of the studied samples (taken under cross-polarized light except for $\mathrm{g}$, which is under plane-polarized light). (a) Beidao granite (TDG-1) showing a hypidiomorphic texture. (b) Sample from the Beidao granite (TDG-2) showing feldspars and quartz with obvious myrmekitic texture associated with perthite. (c) Beidao granite (TDG-3) showing alkali feldspar, biotite, secondary muscovite, and zircon. (d) Beidao granite (TDG-4) showing alkali feldspar altered into the sericite. The large zircon crystal contains melt inclusions. (e) Sample from the Caochuanpu granite (TCG-1) displaying phenocrysts of perthite, plagioclase, and quartz. (f) Sericite alteration in the core of zoned plagioclase (Caochuanpu granite, TCG-2). (g) Biotite grains exhibiting pleochroic haloes around zircons inclusions (Caochuanpu granite, TCG-3). (h) Yuanlongzhen granite displaying general textural features (TYG-1). (i) Yuanlongzhen granite (TYG-3) showing plagioclase, perthite, and quartz. Note some of the fine-grained recrystallized quartz have developed along the edges of the strained quartz. (j) Highly altered plagioclase pseudomorphs associated with biotite that contains tiny zircon inclusions (TYG-6). (k) Interstitial zoned tourmaline in the Roche type rock (RTR-1). (1) Muscovite with abundant fluorapatite inclusions (RTR-2). Abbreviations shown on the photomicrographs are: Afs: Alkali feldspar, Ap: fluorapatite, Bt: biotite, Mc: Microcline, Ms: muscovite, Myr: myrmekite, Pl: plagioclase, Prth: perthite, Qz: Quartz, Ser: sericite, Tnt: titanite, Tur: Tourmaline, and Zrn: Zircon. 
The Caochuanpu granite has an intrusive contact with the Beidao granite in the western Qinling Orogen (Figure 1b). Its fresh color is pink (Figure 2c). The Caochuanpu granite is mega porphyritic in which phenocrysts of alkali felspar, plagioclase, and quartz are embedded in a relatively fine-grained groundmass (Figure 3e). Most of the phenocrysts range in size from 2 to $3 \mathrm{~cm}$. Mineralogically, this granitic is composed of quartz $(25-30 \%)$, perthite $(35-40 \%)$, plagioclase $(24-30 \%)$, biotite $(0.5-2 \%)$, zircon $(0.1-2 \%)$, titanite (up to $1 \%)$, fluorapatite $(0.5-1 \%)$, opaque minerals $(1-2 \%)$, and secondary sericite $(0.5-2 \%)$ (Table 1$)$. Quartz is anhedral to subhedral, whereas the majority of feldspar is euhedral. Alkali feldspar in this granite is perthitic and shows carlsbad twining. Perthite is slightly altered into clays and sericite. Most of the plagioclase phenocrysts are zoned and the core portions are altered into sericite (Figure 3f), suggesting that the granitic magma crystallized due to fractional crystallization.

In most plagioclase crystals, the core is more altered to sericite and clays than the rim, which may be due to the relatively high Ca content found in the inner core (Figure 3f). The common accessory phases found in the Caochuanpu granite are biotite, zircon, titanite, and fluorapatite. The biotite is mostly fresh and contains inclusions of zircon and fluorapatite. Some of the biotite grains are also chloritized. In most of the biotite grains, pleochroic haloes around zircon are common. (Figure 3g).

\subsubsection{Yuanlongzhen Granite and Roche Type Rock}

The Yuanlongzhen granite intrudes into the Paleoproterozoic to Early Paleozoic metamorphic rocks of the Qinling Group and the Early Paleozoic granites (Figure 1b). It is coarse-grained and its fresh color is light pink whereas its weathered color is yellow to reddish-brown (Figure 2d). In this granite, all felsic minerals are distributed homogenously and most of the grains are subhedral to anhedral showing a hypidiomorphic granular texture. Mineralogically, it is composed of quartz $(28-32 \%)$, plagioclase (23-30\%), perthite (20-25\%), microcline (5-10\%), biotite (1-2\%), muscovite (up to $0.5 \%)$, titanite (up to $0.5 \%$ ), zircon ( $0.5-1 \%$ ), rutile (up to $0.5 \%$ ), sericite (up to $2 \%$ ), and opaque minerals (up to $2 \%$ ) (Table 1). Quartz grains are strained are anhedral whereas feldspars grains are euhedral with well-developed faces (Figure 3h). Both feldspars and quartz grains are highly fractured (Figure 3i-k) and their edges reveal recrystallization (Figure 3i). Plagioclase grains are highly altered into sericite and clays (Figure 3j). Most of the alkali feldspar displays carlsbad twinning and is slightly altered into sericite at places. Accessory minerals include biotite, muscovite, titanite, zircon, and rutile with some opaque minerals. Biotite contains abundant zircon inclusions that show pleochroic haloes around (Figure 3j). A few grains of euhedral titanite are also found in the Yuanlongzhen granite (Figure 3i). Modal percentage of minerals from the three granitoid bodies are plotted on the International Union of Geological Sciences (IUGS) classification system [50] of plutonic igneous rocks and all plot in the monzogranite field (Figure 4; Table 1).

The Roche type rock has an intrusive contact with the Beidao granite (Figure 1b). It is coarse-grained and dark gray to black (Figure 2e). It is mainly composed of quartz and black tourmaline (schorl) with minor amounts of muscovite. Traces of zircon, fluorapatite, and rutile are also found as accessory minerals. Tourmaline grains are inhomogeneously distributed and form clusters and separated domains of various extinctions. Texturally, it seems that all the tourmaline grains have been crystallized in the interstitial-free spaces within the quartz aggregates suggest late crystallization of tourmaline than the neighboring quartz or other phases (Figure 3k). Muscovite also contains tiny inclusions of fluorapatite. Mineralogically, the Roche type rock is composed of quartz (60-65\%), tourmaline $(30-36 \%)$, muscovite (1-2\%), zircon (up to $0.5 \%$ ), rutile (up to $0.5 \%$ ), and fluorapatite $(0.5-1 \%$ ) (Table 1 ). 


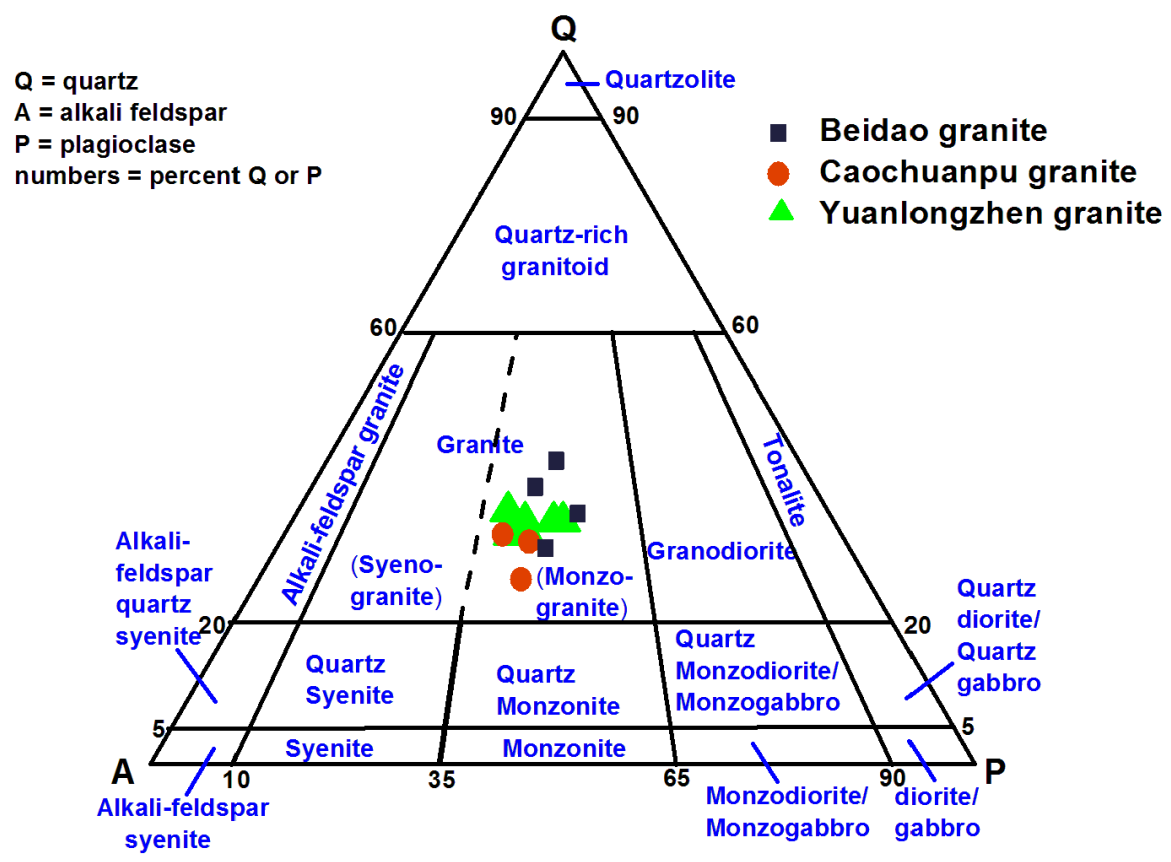

Figure 4. Modal amount (volume \%) from the investigated granite samples from the Tianshui area are plotted in the IUGS classification diagram for plutonic igneous rocks after [50]. Modal abundances were determined using the point-counting method under the optical microscope.

\subsection{Geochemistry}

\subsubsection{Major and Trace Elements Geochemistry}

Major and trace elements chemical compositions of the three granitic bodies and the Roche type rock are given in Table 2. The geochemical results reveal that $\mathrm{SiO}_{2}$ contents in the Beidao granite (samples with titles TDG), Caochuanpu porphyritic granite (TCG), Yuanlongzhen granite (TYG) and the Roche type rock (RTR) vary from 71.99 to $72.61 \mathrm{wt.} \%, 72.33$ to $73.73 \mathrm{wt.} \%, 72.98$ to $74.05 \mathrm{wt}$. \%, and 70.97 to $71.09 \mathrm{wt}$ \%, respectively, all exhibiting a very similar $\mathrm{SiO}_{2}$ range. The $\mathrm{Al}_{2} \mathrm{O}_{3}$ contents in TDG, TCG, TYG, and RTR range from 11.45-12.16 wt. \%, 11.99-12.18 wt. \%, 12.99-13.4 wt. \% and 11.07-12.1 wt. \%, respectively. The $\mathrm{Fe}_{2} \mathrm{O}_{3}$ contents are quite low in the TDG, TCG, TYG, but relatively higher in the RTR samples, exhibiting a range from $0.39-0.78 \mathrm{wt} . \%, 1.16-1.89 \mathrm{wt} . \%, 1.1-1.59 \mathrm{wt}$ \% and 5.96-6.86 wt. \%, respectively. Other major elements also show a narrow range and similar chemical compositions for the three granitoids (see details in Table 2). According to the TAS diagram [51], rocks suites of the Tianshui area plot in the fields of granite to granodiorite (Figure 5). The $\mathrm{Al}_{2} \mathrm{O}_{3} /(\mathrm{CaO}+$ $\mathrm{Na}_{2} \mathrm{O}+\mathrm{K}_{2} \mathrm{O}$ ) vs. $\mathrm{SiO}_{2}$ classification diagram for peraluminous and meta-aluminous [52] as well as I and S-type granites [53] indicate that samples from the Beidao, Caochuanpu, and Yuanlongzhen granites plot in the metaluminous I-type granitoids field. One Yuanlongzhen granite sample (TYG-1) plots in the peraluminous I-type granite field. The Roche type rock samples show a strong affinity towards the peraluminous S-type granitoid field (Figure 6a). 


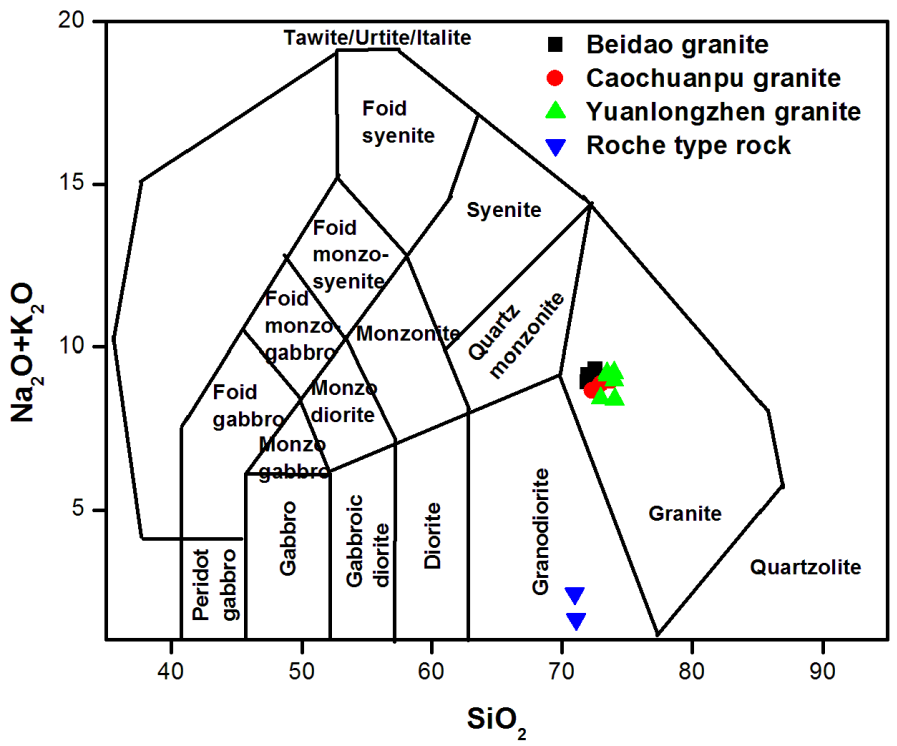

Figure 5. Total alkali-silica (TAS) classification diagram for granitoids. Note all the studied samples from the three granitoids show extremely similar chemical compositions except the Roche type rock that shows low alkali contents, diagram after [51].
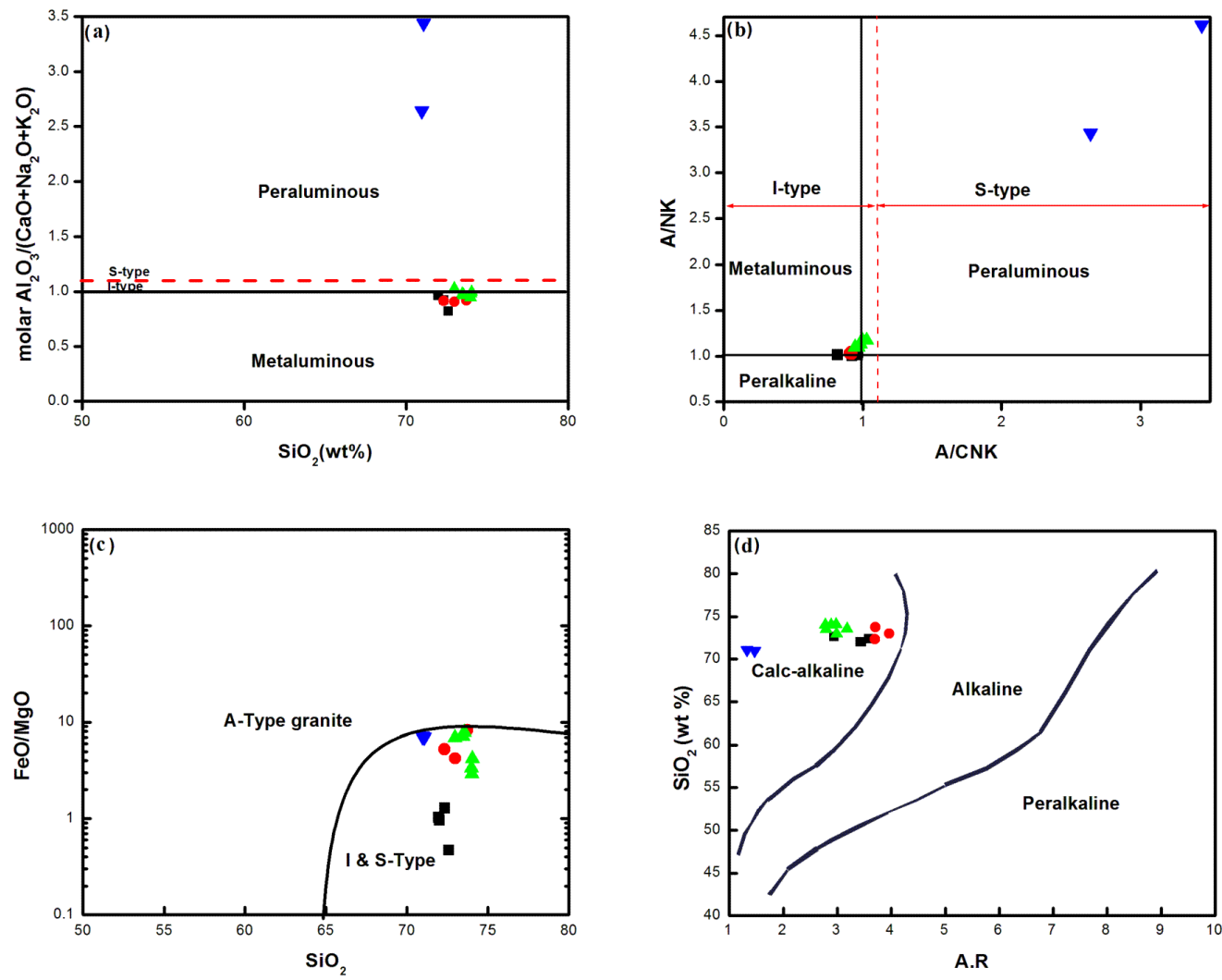

Figure 6. (a) $\mathrm{Al}_{2} \mathrm{O}_{3} /\left(\mathrm{CaO}+\mathrm{Na}_{2} \mathrm{O}+\mathrm{K}_{2} \mathrm{O}\right)$ vs. $\mathrm{SiO}_{2}$ classification diagram for peraluminous and meta-aluminous [52] and I and S-type granites [53]. Solid horizontal and dotted lines indicate a molar alkalinity of 1 and a boundary between I- and S-type granitoids. (b) Alumina index diagram for granitoids and Roche type rock (the boundary between I- and S-type granites are after [54]. Solid vertical and dotted lines mark the boundary between alkalinity of 1 and a boundary between I- and S-type granitoids (c) Data of the Tianshui granitoids and Roche type rock plotted on the $\mathrm{SiO}_{2}$ vs. $\mathrm{FeO} / \mathrm{MgO}$ diagram of [55], indicating I- and S-type origin. (d) $\mathrm{SiO}_{2}$ vs. alkalinity ratio (A.R) diagram after [56] on which all the studied samples plot in the calc-alkaline field. 
Using the Al saturation index, A/CNK diagram (Figure 6b), TDG, TCG, and TYG are classified as I-type whereas RTR is classified as S-type granitoids (Figure 6b). Plots of the Tianshui granitoids and Roche type rock on the $\mathrm{SiO}_{2}$ vs. $\mathrm{FeO} / \mathrm{MgO}$ diagram proposed by [55], show I- and S-type granite field (Figure 6c). The plot of $\mathrm{SiO}_{2}$ against the alkalinity ratio (A.R) [56] reveals calc-alkaline nature for the studied samples, indicating these granitoids (including Roche type rock) formed in a subduction-related environment (Figure 6d).

The $\mathrm{Na}_{2} \mathrm{O} / \mathrm{Al}_{2} \mathrm{O}_{3}$ vs. $\mathrm{K}_{2} \mathrm{O} / \mathrm{A1}_{2} \mathrm{O}_{3}$ diagram (Figure 7a) of [57] reveals that the parental magmas of the Tianshui granitoids and Roche type rock originated from the partial melting of igneous and sedimentary rocks, respectively. The classification diagram of [58] discriminates between magnesian and ferroan granitoids. On the $\mathrm{SiO}_{2}$ vs. $\mathrm{FeO} /(\mathrm{FeO}+\mathrm{MgO})$ diagram, the Beidao granite falls in the magnesian field whereas the Caochuanpu granite and Yuanlongzhen granite show a transition from ferroan to magnesian granitoids. Roche type rock plot in the ferroan field (Figure 7b).

Based on trace element geochemistry, the studied rocks can be discriminated as volcanic-arc to syn-collisional granites when plotted on the $\mathrm{Rb}$ vs. $\mathrm{Y}+\mathrm{Nb}$ (ppm) and $\mathrm{Nb}$ vs. $\mathrm{Y}(\mathrm{ppm})$ discrimination diagrams [59] (Figure 7c,d).
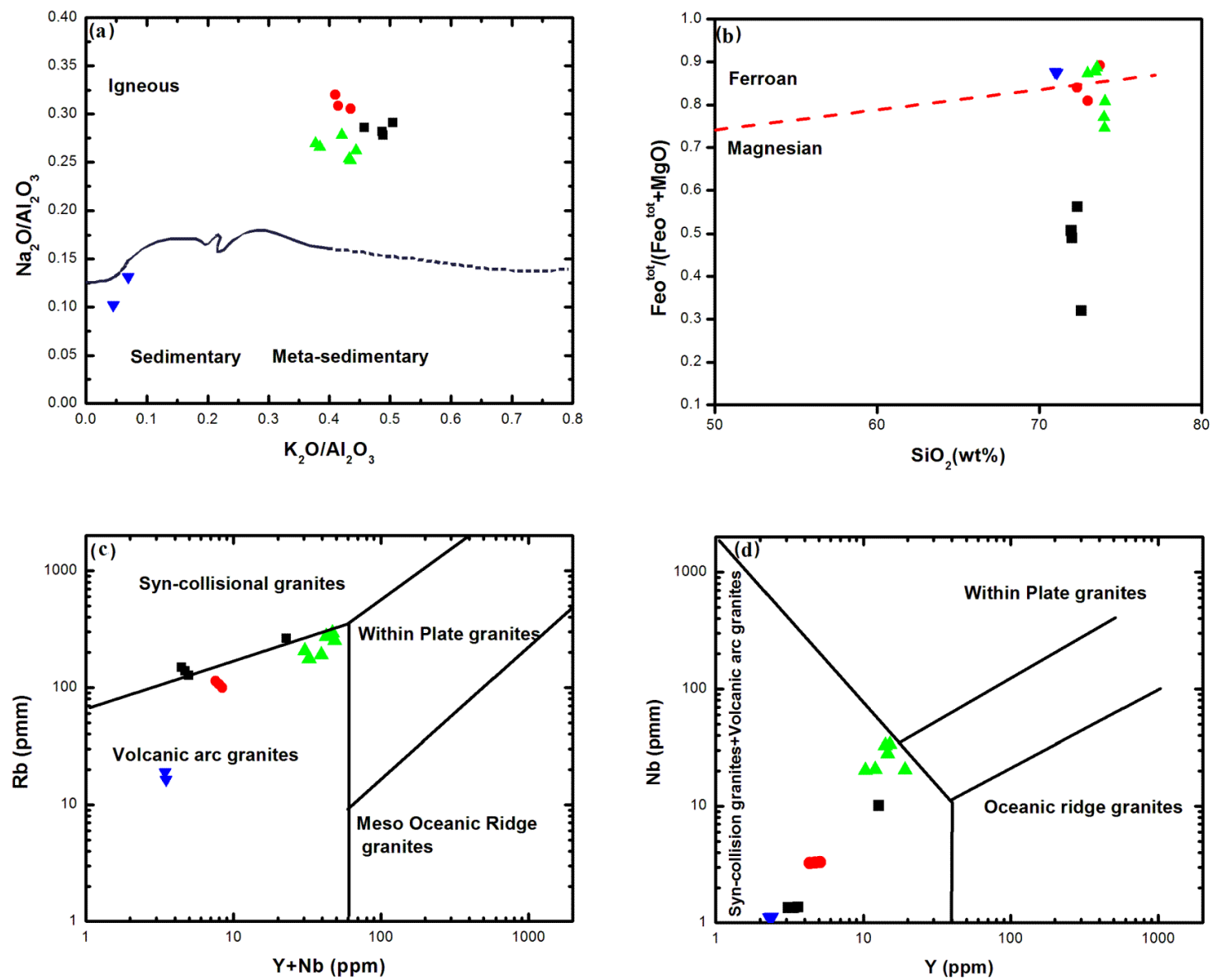

Figure 7. (a) $\mathrm{Na}_{2} \mathrm{O} / \mathrm{Al}_{2} \mathrm{O}_{3}$ vs. $\mathrm{K}_{2} \mathrm{O} / \mathrm{A1}_{2} \mathrm{O}_{3}$ diagram of [57] for the Tianshui granitoids and Roche type rock, (b) position of the granitoids and Roche type rock of the Tianshui area in the $\mathrm{SiO}_{2} \mathrm{vs}$. FeOt/(FeOt $+\mathrm{MgO}$ ) diagram, (c) tectonomagmatic discrimination diagrams of [59] using $\mathrm{Y}+\mathrm{Nb}$ (ppm) vs. $\mathrm{Rb}$ (ppm) and (d) Y (ppm) vs. $\mathrm{Nb}(\mathrm{ppm})$.

\subsubsection{Rare Earth Elements Geochemistry}

Rare earth elements (REE) data of the three granitic bodies and the Roche type rock are presented in Table 1 and the chondrite-normalized [60] values are plotted in Figure 8a. Granitoid samples from the three locations (TDG, TCG, and TYG) are almost similar and also similar to the chondrite-normalized 
REE patterns of the Bulk Continental Crust reported by [61]. The REE values of Roche type rock are much depleted and are distinct from the other granitoid rocks of the Tianshui area. The REE profiles indicate that the magma of these rocks was likely originated from the partial melting of continental crust. Negative Eu anomalies in all granite samples from the Caochuanpu and Yuanlongzhen granites and the Roche type rock indicate depletion of magma due to the removal or fractionation of apatite and plagioclase in the crystallization process. In contrast, three samples from the Beidao granite (TDG-1, TDG-2, and TDG-3) show positive Eu anomalies, suggesting the enrichment of magma due to the addition or accumulation of apatite and plagioclase during their magmatic crystallization. The presences of both positive and negative anomalies in the Beidao granite indicate a complex or diverse origin of the magma. Chondrite-normalized REE data slope gently to the right indicating a clear differentiation trend between LREEs and HREEs, as represented by the enrichment and depletion in LREEs and HREEs, respectively. The Primitive mantle-normalized spider plots also show slightly positive crests to strongly negative troughs for $\mathrm{Eu}\left(\mathrm{Eu} / \mathrm{Eu}^{*}=0.18\right.$ to 1.20$)$ for the Beidao granite whereas the Caochuanpu and the Yuanlongzhen granites and the Roche type rock display moderate to strongly negative $\mathrm{Eu}$ anomalies $\left(\mathrm{Eu} / \mathrm{Eu}^{*}=0.29\right.$ to 0.52$)$. Most of the granitoids show positive Ce anomalies $\left(\mathrm{Ce} / \mathrm{Ce}^{*}=0.98\right.$ to 1.69$)$ (Table 2$)$. The LREE/HREE and $(\mathrm{La} / \mathrm{Yb})_{\mathrm{N}}$ ratios are relatively high in samples of the Beidao granitoids as compared to the other two types of granites and the Roche type rock (Table 2), indicating the nature of the magma for Beidao was different than the other rock types.
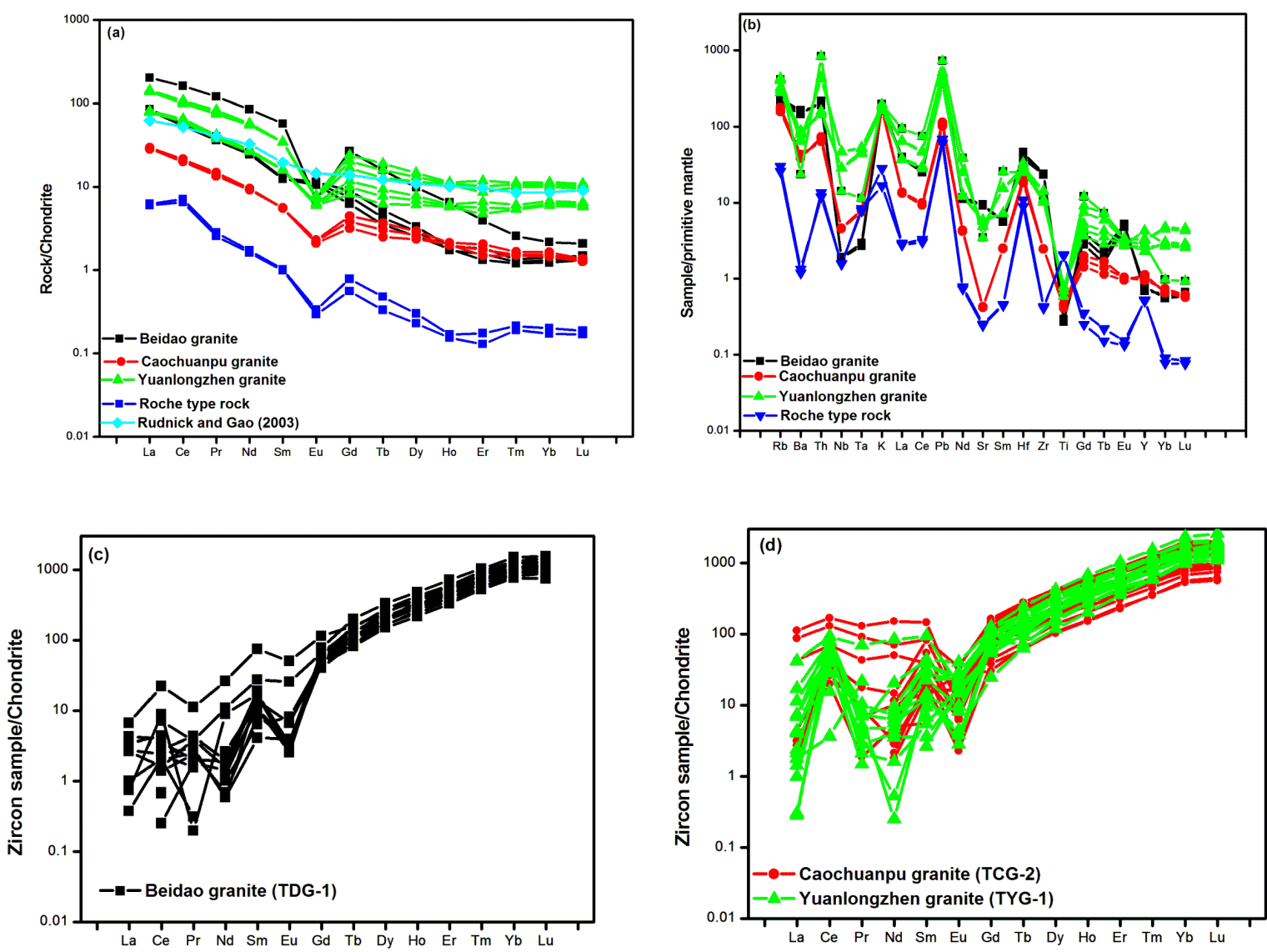

Figure 8. (a) Chondrite-normalized rare earth element (REE) diagram for the analyzed samples. (b) Primitive mantle-normalized diagram for the analyzed samples. Normalizing values for REE and trace elements are from [62]. (c) Chondrite-normalized REE patterns of the dated zircons from the Beidao granite. (d) Chondrite-normalized REE patterns of the dated zircons from the Caochuanpu and the Yuanlongzhen granites. 
The three granitoid bodies generally display enrichment in $\mathrm{K}, \mathrm{Rb}, \mathrm{Th}, \mathrm{Pb}$ and $\mathrm{Hf}$, and depletion in $\mathrm{Ba}, \mathrm{Nb}, \mathrm{Ce}, \mathrm{Sr}$ (except three samples from the Beidao granite that have high $\mathrm{Sr}$ contents), Ti (except Roche type rock having high $\mathrm{Ti}$ ), Ta and $\mathrm{Y}$ (except Roche type rock having high $\mathrm{Y}$ content) and $\mathrm{Yb}$ that show spikes on the primitive mantle-normalized spider diagram (Figure 8b). These features indicate the differentiation of minerals (such as feldspar, fluorapatite, and rutile) in the magma. In all samples, $\mathrm{Nb}$ and $\mathrm{Ta}$ are depleted compared to $\mathrm{La}$ and $\mathrm{K}$, thus confirming a subduction-related tectonic environment. The enrichment of $\mathrm{Sr}$ and $\mathrm{Eu}$ in the Beidao granites indicates the accumulation of Ca-rich plagioclase, whereas enrichment of $\mathrm{Ti}$ in the Roche type rock indicates the accumulation of rutile during magmatic crystallization. Moreover, the deficiency of $\mathrm{Nb}$ and $\mathrm{Ta}$ in the studied samples is a significant characteristic of magma that can be derived from the continental crust and calc-alkaline plutonic source formed in a subduction regime [63].

\subsection{U-Pb Isotope Ratios and Trace Element Composition of Zircons}

Zircons from Beidao (sample: TDG-1), Yuanlongzhen (sample: TYG-1), and Caochuanpu (sample: TCG-2) granites were analyzed for U-Pb isotope ratios and trace element contents. Zircon grains from all three granitoid bodies in the cathodoluminescence pictures show growth (oscillatory) zoning, columnar, prismatic shapes, and the grains range in size from 100 to $350 \mu \mathrm{m}$. A few zircon grains also show mineral inclusions (Figure 9).

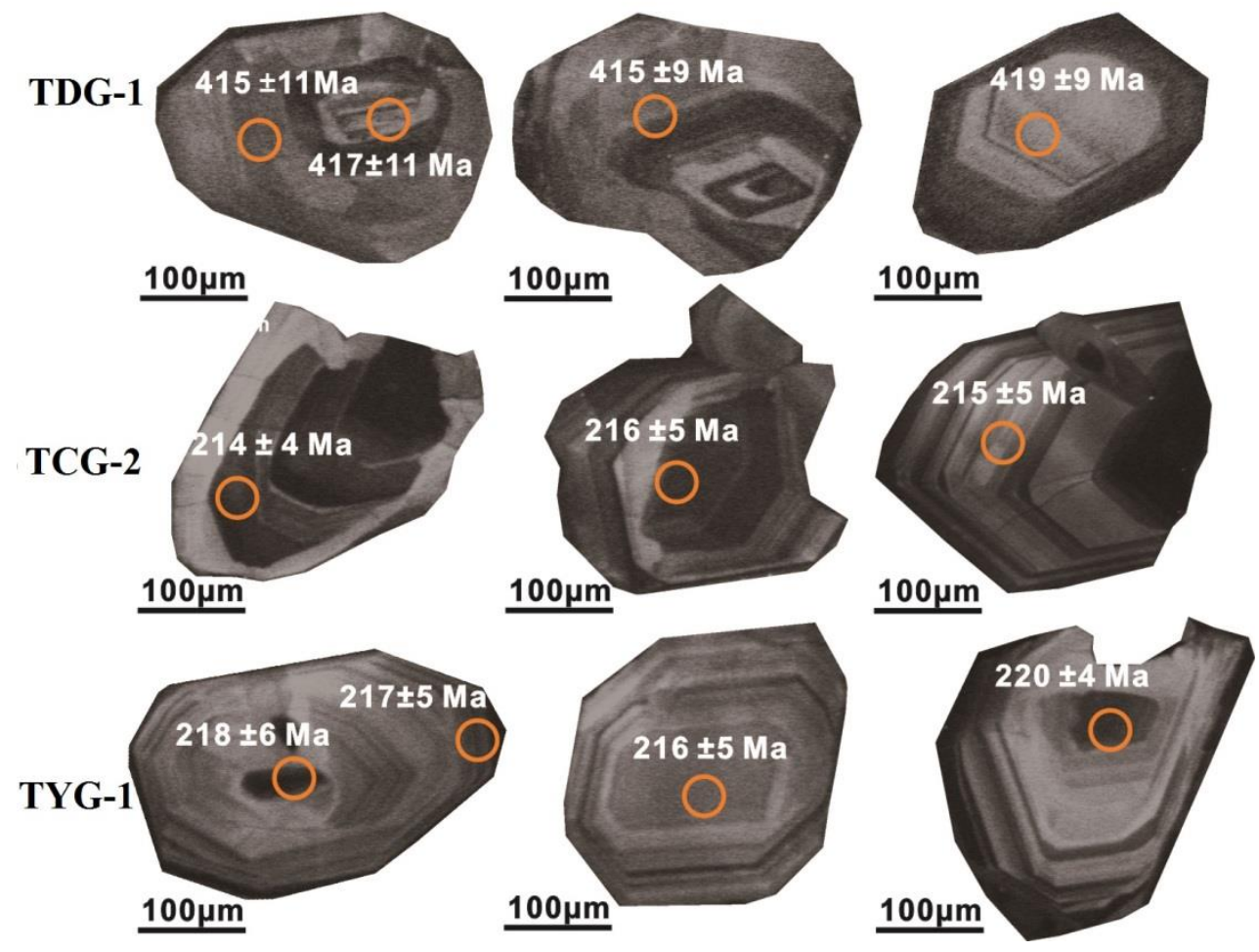

Figure 9. Cathodoluminescence (C) images of representative zircons grains analyzed from Beidao (TDG-1), Caochuanpu (TCG-2), and Yuanlongzhen (TYG-1) granites. Circles with digits on zircon grains represent Laser Ablation Multicollector Inductively Coupled Plasma Mass Spectrometry (LA-MC-ICPMS) analyses and age values, respectively.

The $\mathrm{U}-\mathrm{Pb}$ isotope ratios and age data from the analyzed zircons are shown in Table 3 and the trace element contents are presented in Table 4 . Zircons from the three granites yielded weighted mean ${ }^{206} \mathrm{~Pb} /{ }^{238} \mathrm{U}$ ages of $417 \pm 5(\mathrm{MSWD}=0.089)$ for Beidao, $219 \pm 3(\mathrm{MSWD}=0.21)$ Ma for Yuanlongzhen, and $216 \pm 3($ MSWD $=0.29)$ for Caochuanpu granites (Figure 10; Table 3). The data plotted on the concordia diagrams have concordance $>95 \%$, except a few data points that had a relatively lower concordance rate (Table 3). Degree of concordance is calculated as $\left[1 /\left(1-{ }^{206} \mathrm{~Pb} /{ }^{238} \mathrm{U}\right.\right.$ age $/{ }^{207} \mathrm{~Pb} /{ }^{235} \mathrm{U}$ 
age) $\times 100$ ]. The Yuanlongzhen and Caochuanpu granites are intrusive into the Beidao granite, which is confirmed by their young ages.
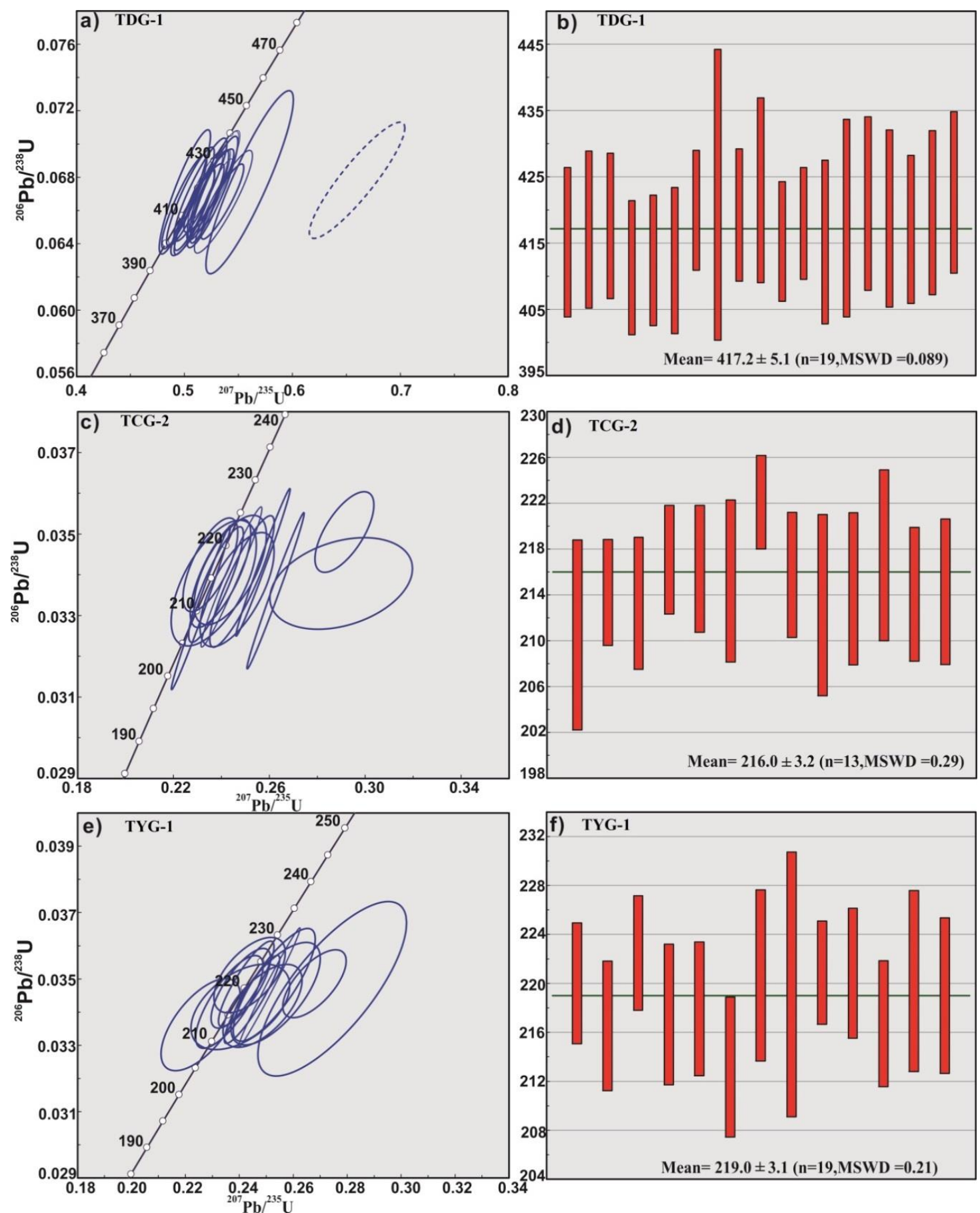

Figure 10. U-Pb zircon concordia diagrams $(\mathbf{a}, \mathbf{c}, \mathbf{e})$ and their weighted mean values $(\mathbf{b}, \mathbf{d}, \mathbf{f})$ for Beidao (TDG-1), Caochuanpu (TCG-2), and Yuanlongzhen (TYG-1), granites, respectively.

Our newly obtained U-Pb zircon age data indicate that the magma of these older (e.g., Beidao) and younger (e.g., Caochuanpu and Yuanlongzhen) granites formed during Early Devonian (Lochkovian) and Late Triassic (Norian) due to the subduction of the oceanic lithosphere from the proto-Tethyan (Figure 11a) and the Paleo-Tethyan Qinling (Figure 11b), respectively. 
(a)

\section{Early Devonian}

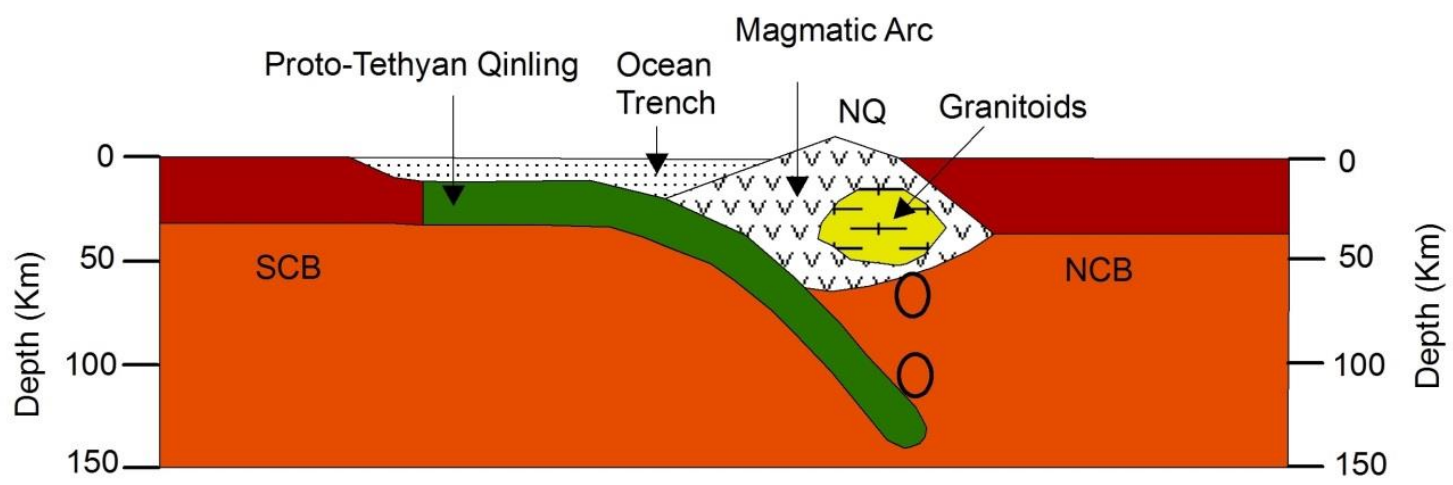

(b)

\section{Late Triassic}

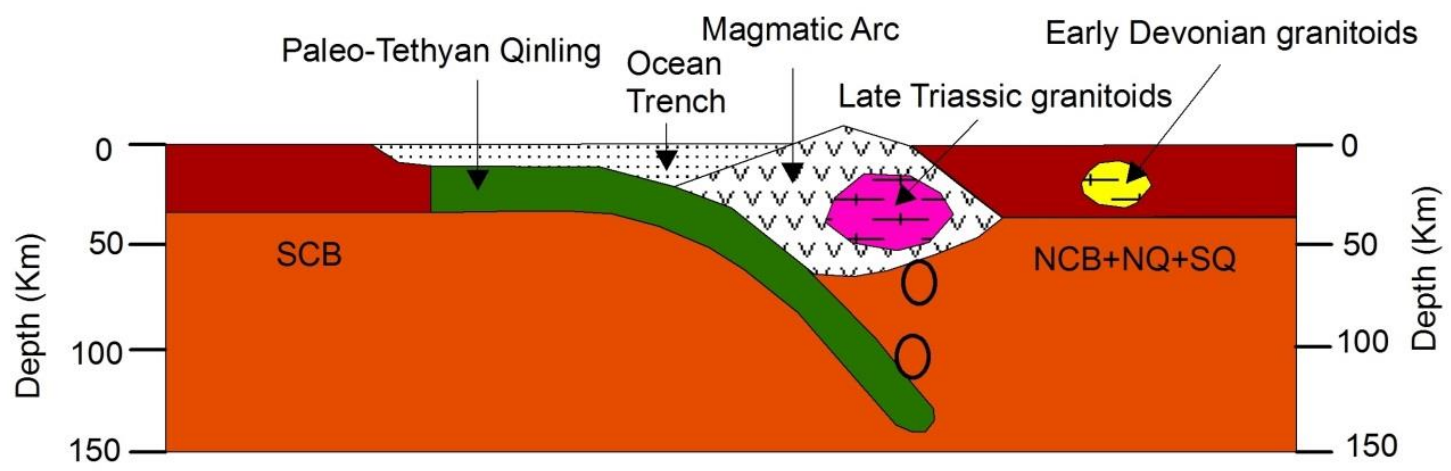

Figure 11. A schematic model that describes the subduction of the Proto-Tethyan Ocean (a) and Paleo-Tethyan ocean $(\mathbf{b})$ that produced the granitoids of the Tainshui area. Abbreviations are; $\mathrm{NCB}=$ North China block; SCB = South China block; NQ = North Qinling orogen; SQ = South Qinling orogen.

Based on trace element geochemistry, it can be stated that the majority of zircon grains from the three granitoids display slightly different geochemical characters in the chondrite normalized REE diagrams (values from [60]) (Figure 8c,d). Total REE contents of zircons of the Early Devonian Beidao granite (TDG-1) range from 368 to 759 ppm (Table 4), whereas U and Th content vary from 500 to 1043 and 47 to $169 \mathrm{ppm}$, respectively (Table 3). The Th/U ratios vary between 0.09 and 0.22 with a mean ratio of 0.14 (Table 3), indicating their magmatic source, similar to the Marid zircons of South Africa [64]. Total REE contents of zircons o the Late Triassic Yuanlongzhen granite (TYG-1) range from 495 to 1197 ppm (Table 4), whereas U and Th contents vary from 235 to 712 and 89 to 485 ppm, respectively (Table 3). The Th/U ratios scatter between 0.52 and 0.90 (Table 3) with a mean ratio of 0.68 . Total REE contents of zircons of the Late Triassic Caochuanpu granite (TCG-2) spread between 274 and 1026 ppm (Table 4), whereas $U$ and Th contents vary from 154 to 1333 and 81 to 689 ppm, respectively (Table 3). The Th/U ratios vary from 0.40 to 0.78 (Table 3 ) with a mean ratio of 0.53 . Chondrite-normalized REE patterns of the analyzed zircons display downward slopes to the left (Figure 8c,d), showing an LREE depletion and HREE enrichment during their crystallization.

Trace and REE data from the studied zircons in all the three granites, their oscillatory zoning, and relatively higher $\mathrm{Th} / \mathrm{U}$ ratios $(>0.5)$ indicate their magmatic origin without any metamorphic growth 
or overprint. A positive Ce anomaly in the studied zircons further attests the substitution of $\mathrm{Ce}^{3+}$ to $\mathrm{Ce}^{4+}$ in the zircon lattice whereas the negative Eu anomaly shows their crystallization from a magma that was simultaneously crystallizing Ca-rich plagioclase.

\section{Discussion}

It is widely considered that the QOB formed as a result of the Caledonian, Hercynian, and Indosinian orogenies [1,65,66] between 440-400 Ma, 340-260 Ma, and 255-210 Ma, respectively [66, 67]. Orogeny occurs when tectonic plates collide, which results in widespread deformation, often accompanied by granite intrusion and metamorphism [68]. Granitic intrusions are formed due to orogenic and anorgenic processes [69]. Orogenic granites are generally subdivided into M-, I-, and S-type granites.

The Beidao granite is classified as calc-alkaline, metaluminous I- type monzo-granite (Figures 4 and 6). Its position in the $\mathrm{Y}+\mathrm{Nb}(\mathrm{ppm}) \mathrm{vs}$. $\mathrm{Rb}(\mathrm{ppm})$ and $\mathrm{Y}(\mathrm{ppm}) \mathrm{vs}$. $\mathrm{Nb}$ (ppm) tectonomagmatic discrimination diagrams [59] suggests that it formed in a syn-collision tectonic setting. The general models for the origin of I-type granite are the mixing of two kinds of magma, basaltic and acid magmas generated by partial melting of the upper mantle and lower crust, respectively [15,30,70-73] and/or by partial melting of igneous parental material from either the subducting oceanic plate or the overlying mantle wedge. Typically the latter have high concentrations of $\mathrm{Na}_{2} \mathrm{O}$ and $\mathrm{CaO}$ [52]. The Beidao granite has high concentrations of $\mathrm{Na}_{2} \mathrm{O}(>3.2 \%)$ but relatively low concentrations of $\mathrm{CaO}(<1.58 \%)$, which may suggest that the Beidao granite is hybrid and formed as a result of mixing of two magmas from diverse sources, e.g., mantle and crust. The meta-aluminous nature of the granite shows that its magma was possibly derived from basic rocks (melting of the basement amphibolites) [74,75]. This would point to the lower crust as a source for the Beidao granite. The Beidao granite is further classified as magnesian granite (Figure $7 \mathrm{~b}$ ) and can be linked to relatively oxidizing conditions and hydrous magmas [76]. Melts from the basaltic lower crust are commonly characterized by low $\mathrm{Mg \#}(<40)$, whereas those with $\mathrm{Mg \#}>40$ can only be formed by the involvement of mantle components [77,78]. Note that all the samples of the Beidao granite show high Mg\# values (over 40; Table 2), which suggests the participation of mantle components. Therefore, magma mixing was probably involved in the evolution of the Beidao granite, or initially magma was formed from partial melting of subducting oceanic lithosphere with large contributions of mantle components that assimilated with the rocks of the continental crust. The U-Pb zircon dating shows that the Beidao granite crystallized at $417 \pm 5 \mathrm{Ma}$ (Figure 10). Since the Caledonian orogeny in the NQB occurred during 400-500 Ma [79], its crystallization can be related to the Caledonian orogeny. The age of the Beidao granite is consistent with the U-Pb of the granitoid body of the Niutou River located in the western segment of the NQB [80]. The granitoid body of Niutou River, however, is a peraluminous calc-alkaline I-type granite and has moderately negative $\mathrm{Eu}$ anomalies [80]. In contrast, the Beidao granite is a metaluminous I-type granite (Figure 6a,b) and has positive to negative Eu anomalies. The positive Eu anomaly suggests accumulation of Ca-rich plagioclase during magma crystallization, whereas the negative Eu anomaly is indicative of the removal of Ca-rich plagioclase during magma crystallization. The positive Eu anomaly may indicate that the parental magma was derived by partial melting of tonalitic rocks in the lower crust $[81,82]$. In addition, deficiency in the $\mathrm{Nb}$ and $\mathrm{Ta}$ is a significant characteristic of magma derived from the continental crust and calc-alkaline plutonic rocks formed in a subduction regime [63].

The Beidao granite is also contemporaneous with the Damaogou granite (U-Pb zircon age $420 \pm 1$ Ma [19]), Yanwan intrusion (U-Pb zircon age $414 \pm 2 \mathrm{Ma}$ [83]) and the lower limit of the emplacement age of the Huichizi granite complex (462 to $418 \mathrm{Ma}$ [18]). The metamorphic ages (413 $\pm 1 \mathrm{Ma})$ in the Honghuapu subduction-related intrusion, determined by LA-ICPMS zircon U-Pb dating method, can accurately constrain the end of the Caledonian collisional orogeny in the western segment of NQB [84]. As the Beidao granite was intruded into the Qinling Group of the QC, the QC is considered as a part of the Caledonian orogeny [85], and also considered as the Caledonian orogenic belt [10]. Regional metamorphism in QC, which consisted of early eclogite facies of ca. $505 \mathrm{Ma}$, subsequent granulite 
facies (HP granulite facies of ca. $500 \mathrm{Ma}$ followed by LP granulite facies of ca. $450 \mathrm{Ma}$ ), and final amphibolite facies metamorphism of ca. $420 \mathrm{Ma}$, occurred in the Early Paleozoic [86]. In the NQB, the QC suffered intensive and widespread migmatization, and the metamorphic zircons of the QC give $\mathrm{U}-\mathrm{Pb}$ ages of ca. 420-400 Ma, which can constrain the time of decompression of the mafic lithologies (amphibolite facies rocks) in this region [39]. The thermal event possibly formed the Beidao granitoids in the syn- to post-collision tectonic settings.

During Late Proterozoic to Early Paleozoic time, a Proto Tethyan Qinling ocean existed between the NCB and SCB [87]. Since the Late Proterozoic, the stratigraphy of the two blocks has been completely different, indicating that the two blocks were separated. The Proto Tethyan Qinling Ocean was closed due to Ordovician subduction, which led to the development of the arc trench system and back-arc basin in the NQB. The Middle Paleozoic subduction and collision along the Shangdan suture accreted the SCB to the NCB, leading to extensive Caledonian metamorphism, magmatism, and tectonic deformation $[12,88]$. The subduction-related granitoids and calc-alkaline mafic magmatism largely in the NQB occurred at ca. 440-400 Ma [89-93]. The Shangdan Suture Zone (SSZ) (Figure 1a) provides compelling evidence for the closing of the Proto Tethys Qinling Ocean. The SSZ (Figure 1), which contains an ophiolite mélange comprised of ultra-mafic to mafic rocks and radiolarian cherts $[13,94]$, divides the NQB from the SQB and is usually thought to delineate the main place of the collision and subduction events that headed the amalgamation of the NCB and the SCB $[8,94,95]$. The Proto-Tethys Ocean was gradually closed between $480-400 \mathrm{Ma}$, indicating one global event of continental assembly [96]. Therefore, it is inferred that the Beidao granite was formed due to the subduction of the Proto-Tethys Qinling Ocean under the NCB (Figure 11a).

The Caochuanpu and the Yuanlongzhen monzo-granites, characterized as metaluminous I-type and metaluminous to very slightly peraluminous I-types, respectively (Figure 6a,b), suggest that their magmas were either generated by melting of amphibolites or derived from partial melting of preexisting of basic rocks [74,75]. However according to [97], the magma of peraluminous rocks usually results in collision zones due to the partial melting of sedimentary/metasedimentary rocks. The assimilation of metasedimentary rocks in the magma may lead to the slightly meta-aluminous nature of the granitoids [58]. The magma of these calc-alkaline granites (Figure 6d) was possibly derived by the partial melting of an igneous protolith (Figure 7a) in syn-collision or a volcanic arc tectonic setting as shown by the $\mathrm{Y}+\mathrm{Nb}(\mathrm{ppm}) \mathrm{vs}$. $\mathrm{Rb}(\mathrm{ppm})$ and $\mathrm{Y}(\mathrm{ppm}) \mathrm{vs} . \mathrm{Nb}(\mathrm{ppm})$ plots on the tectonomagmatic discrimination diagrams [59]. According to Chappel and White [52], I-type granites are formed through the partial melting of an igneous parental material either from a subducting oceanic plate or from the overlying mantle wedge. That is why they are characterized by high concentrations of $\mathrm{Na}_{2} \mathrm{O}$ and $\mathrm{CaO}$. The relatively higher concentrations of $\mathrm{Na}_{2} \mathrm{O}(>3.2 \%)$ but relatively lower concentrations of $\mathrm{CaO}(<1.06 \%)$ in these granites suggest a hybrid source that likely formed due to the mixing of two magmas of diverse sources, e.g., mantle and crust.

The transition between ferroan to magnesian granitoids (Figure $7 \mathrm{~b}$ ) could be linked with the presence of limited $\mathrm{H}_{2} \mathrm{O}$ and low oxygen fugacity in the partial melting zone [58] and the conditions of anhydrous silicate melt crystallization. In contrast, the magnesian granitoids indicate relatively oxidizing conditions and hydrous magmas as proposed by Frost [76]. Therefore, it seems that these granitoids crystallized from a magma with the intermediate conditions relative to these factors. The lower Mg\# (22-35, mean 32; Table 2) of the Caochuanpu granite suggests it originated from a crust-derived magma without any involvement of the mantle components. Similarly, the relatively lower Mg\# values (23-44, mean 29; Table 2) of the Yuanlongzhen granite also suggest its origin from a crust-derived magma with almost no involvement of the mantle components. The REE data provide further evidence that the source magma was likely formed by the partial melting of lower continental crust (Figure 7a,b). Moreover, the deficiency of $\mathrm{Nb}$ and $\mathrm{Ta}$ in the Caochuanpu and the Yuanlongzhen granites indicates their magma was possibly derived from the melting of continental crust and/or calc-alkaline rocks that usually form in subduction-related tectonic regimes as observed in rocks from elsewhere [63]. We propose a geodynamic model for the Tianshui granitoids that is supported 
by petrological ideas and observations. The rising and underplating of mafic magma derived from the mantle wedges may lead to melting the lower continental crust to produce granitic magmas. The dehydration of oceanic crust could efficiently reduce the solidus of the mantle wedge leading in the generation of mafic magma [98] when the oceanic lithosphere of the Paleo-Tethyan Qinling Ocean subducted under the accreted NCB, North Qinling and South Qinling plates (Figure 11b). The underplating, ascent, and extraction of mantle wedge mafic magmas can melt the lower continental crust and produce parental magma for the Tianshui granitoids.

The origin of the Roche type rock is debatable since it involves complex processes. Its origin was previously ascribed from various sources including that of magmatic [99-103] and/or metasomatic/hydrothermal [102]. The petrographic and geochemical study from the Roche type rock suggests that this rock could be formed by hydrothermal solutions as the product of these hot solutions charged with boron leftover from the magmatic phase during the crystallization of granites. The assimilation of sedimentary sequences at the upper surfaces of the subducting slabs could contaminate the magmas to produce Roche type rocks.

The magmatism during the Triassic (216 and 219 Ma of Caochuanpu and Yuanlongzhen granites) and other similar age (ca. 225-185 Ma) magmatic rocks in the area [2] suggest that these granites formed in the QOB by the northward subduction of the Paleo-Tethyan oceanic crust under the continental plate. Palaeomagnetic and geological data also support the closure of the Paleo-Tethys Ocean during the Triassic period [104]. Before that the Indosinian orogeny caused the Early Mesozoic (245-205) granitoid magmatism in the QOB [105]. Therefore, the Tianshui granitoids represent a collage of the rocks that were associated with the post-collisional regional-scale amphibolite facies metamorphism that occurred at the end of the so-called Caledonian orogeny in the Paleozoic, followed by the Indosinian orogeny in the Mesozoic. Looking at the regional structures, the extensional setting can be envisaged by the South Qinling Orogen, which is parallel to the north passive continental margin of the SCB. The rift-related Ordovician to Silurian alkaline magmatism and sedimentation occurred on the south margin of the Southern Qinling Orogen [106] inferring that a rift system or fault valley system was established in the region. Simultaneously during the Devonian convergence along the SSZ, the southern edge of the South Qinling Orogen progressively separated from the SCB and developed a basin during the Carboniferous and Permian periods known as the Paleo Tethyan Qinling Ocean (Figure 11b). During the Carboniferous to Early Triassic, the South Qinling orogenic belt was separated from the SCN by this ocean. Subduction of the Paleo Tethyan Qinling Ocean was initiated in the Early Triassic, which produced island-arc type calc-alkaline and volcanic rocks [107] along with calc-alkaline granitoids (Figure 11b). During the Triassic, the orogenic process produced the Caochuanpu and Yuanlongzhen along the SSZ. This magmatic activity could be probably linked to the intracontinental collisions and thickening of the crust caused by the northward movement of the SCB. The Triassic granitoids in the QOB were thus developed in a continental arc setting, preceding the final closure of the Paleo Tethyan Qinling Ocean between the Yangtze and North China continental plates [16,108,109].

The Mianlüe Suture Zone (Figure 1a) also provides evidence for the closure of the Paleo Tethyan Qinling Ocean [8]. The MSZ contains broken fragments of ophiolite sequences i.e., gabbros, ultramafic rocks, oceanic tholeiites, and overlying radiolarian cherts $[8,10]$. The MSZ can be related to the Indosinian Orogeny on the Asian plate $[2,8,10,14,88]$. Therefore, we infer that the Caochuanpu and Yuanlongzhen granites of the Tianshui area are formed during the Indosinian Orogeny during Mesozoic times as a result of the subduction of the oceanic lithosphere.

\section{Conclusions}

The following conclusions are drawn based on the petrographic, geochemical, and geochronological data:

(1) The Tianshui granitoids (Beidao, Caochuanpu, and Yuanlongzhen) are monzogranites.

(2) Their $\mathrm{Al}$ saturation index $\left(\mathrm{A} / \mathrm{CNK}\right.$ vs. $\left.\mathrm{SiO}_{2}\right)$ scatter around the boundary of metaluminous to peraluminous granites. 
(3) Geochemical data further classify them as magnesian (Beidao granite), ferroan to magnesian types (Caochuanpu and Yuanlongzhen). The three types granites likely formed in subduction-related tectonic settings and their magmas were formed due to the partial melting of igneous protoliths.

(4) On the tectonic classification diagrams, all the granites plot in the fields of volcanic arc granites + syn-collisional granites. $\mathrm{SiO}_{2}$ vs. $\mathrm{FeO} / \mathrm{MgO}$ diagram points towards I-type for the three granitoid bodies. The REE data, additionally, confirms their magma formed by the partial melting of the lower continental crust.

(5) The weighted mean ${ }^{206} \mathrm{~Pb} /{ }^{238} \mathrm{U}$ zircon ages of $417 \mathrm{Ma}$ for the Beidao granite can be linked to the syn- to the post-collisional tectonic setting, showing contemporaneous age data with the regional-scale amphibolite facies metamorphism that accrued at the end of the so-called Caledonian Orogeny in the QOB.

(6) The magmatism (219 to $216 \mathrm{Ma}$ ) of the Yuanlongzhen and Caochuanpu granites is the result of the Mesozoic (245-205 Ma) Indosinian Orogeny related to the subduction of the oceanic lithosphere.

Author Contributions: Conceptualization, M.S.M. and C.Z.; methodology, M.S.M. and C.Z.; software, M.S.M., A.H., D.D., M.S.B., M.B., J.Z., Q.Z. and S.A.H.; validation, M.S.M., C.Z., A.H., H.U.R., D.D., M.S.B., M.B., J.Z., Q.Z. and S.A.H.; formal analysis, M.S.M., A.H., D.D., J.Z. and Q.Z.; investigation, M.S.M. and C.Z.; resources, C.Z.; data curation, M.S.M., A.H., D.D., J.Z., Q.Z. and S.A.H.; writing-original draft preparation, M.S.M.; writing-review and editing, M.S.M., C.Z. and H.U.R.; visualization, M.S.M., C.Z., M.S.B. and M.B.; supervision, C.Z.; project administration, C.Z.; funding acquisition, C.Z. All authors have read and agreed to the published version of the manuscript.

Funding: This work was financially supported by the Research Funds by NSFC (No. 41571177) and the Fundamental Research Funds for the Central Universities (No. lzujbky-2017-it26).

Acknowledgments: The authors are grateful to two anonymous reviewers for their useful suggestions and critical comments that improved the manuscript significantly.

Conflicts of Interest: The authors declare no conflict of interest.

\section{References}

1. Mattauer, M.; Matte, P.; Malavieille, J.; Tapponnier, P.; Maluski, H.; Qin, X.Z.; Lun, L.Y.; Qin, T.Y. Tectonics of the Qinling belt: Build-up and evolution of eastern Asia. Nature 1985, 317, 496-500. [CrossRef]

2. Zhang, G.; Zhang, B.; Yuan, X.C.; Xiao, Q. Qinling Orogenic Belt and Continental Dynamics; Science Press: Beijing, China, 2001.

3. Dong, Y.; Zhang, G.; Neubauer, F.; Liu, X.; Genser, J.; Hauzenberger, C. Tectonic evolution of the Qinling orogen, China: Review and synthesis. J. Asian Earth Sci. 2011, 41, 213-237. [CrossRef]

4. Wu, Y.-B.; Zheng, Y.-F. Tectonic evolution of a composite collision orogen: An overview on the Qinling-Tongbai-Hong'an-Dabie-Sulu orogenic belt in central China. Gondwana Res. 2013, 23, 1402-1428. [CrossRef]

5. Wang, R.; Xu, Z.; Santosh, M.; Liang, F.; Fu, X. Petrogenesis and tectonic implications of the Early Paleozoic intermediate and mafic intrusions in the South Qinling Belt, Central China: Constraints from geochemistry, zircon U-Pb geochronology and Hf isotopes. Tectonophysics 2017, 712, 270-288. [CrossRef]

6. Chen, L.; Yan, Z; Guo, X.; Fu, C. Melting of the Meso-Neoproterozoic juvenile crust for the origin of the Late Triassic Mo mineralization in South Qinling, central China: Evidence from geochronology and geochemistry of the Yangmugou deposit. J. Asian Earth Sci. 2019, 174, 109-125. [CrossRef]

7. Kröner, A.; Zhang, G.; Sun, Y. Granulites in the Tongbai area, Qinling belt, China: Geochemistry, petrology, single zircon geochronology, and implications for the tectonic evolution of eastern Asia. Tectonics 1993, 12, 245-255. [CrossRef]

8. Meng, Q.-R.; Zhang, G.-W. Timing of collision of the North and South China blocks: Controversy and reconciliation. Geology 1999, 27, 123-126. [CrossRef]

9. Dong, Y.; Zhang, X.; Liu, X.; Li, W.; Chen, Q.; Zhang, G.; Zhang, H.; Yang, Z.; Sun, S.; Zhang, F. Propagation tectonics and multiple accretionary processes of the Qinling Orogen. J. Asian Earth Sci. 2015, 104, 84-98. [CrossRef]

10. Dong, Y.; Santosh, M. Tectonic architecture and multiple orogeny of the Qinling Orogenic Belt, Central China. Gondwana Res. 2016, 29, 1-40. [CrossRef] 
11. Wang, X.; Wang, T.; Zhang, C. Granitoid magmatism in the Qinling orogen, central China and its bearing on orogenic evolution. Sci. China Earth Sci. 2015, 58, 1497-1512. [CrossRef]

12. Li, Y.; Liang, W.; Zhang, G.; Ran, Y.; Shen, Q.; Wang, J.; Jin, C. Granitoid emplacement during syn-convergent transtension: An example from the Huamenlou pluton in North Qinling, central China. Geosci. Front. 2018, 9, 191-205. [CrossRef]

13. Meng, Q.-R.; Zhang, G.-W. Geologic framework and tectonic evolution of the Qinling orogen, central China. Tectonophysics 2000, 323, 183-196. [CrossRef]

14. Pei, X.; Li, Y.; Lu, S.; Chen, Z.; Ding, S.; Hu, B.; Li, Z.; Liu, H. Zircon U-Pb ages of the Guanzizhen intermediate-basic igneous complex in Tianshui area, West Qinling, and their geological significance. Geol. Bull. China 2005, 24, 23-29.

15. Zhang, H.; Jin, L.; Zhang, L.; Nigel, H.; Zhou, L.; Hu, S.; Zhang, B. Geochemical and Pb-Sr-Nd isotopic compositions of granitoids from western Qinling belt: Constraints on basement nature and tectonic affinity. Sci. China Ser. D Earth Sci. 2007, 50, 184-196. [CrossRef]

16. Yanjing, C. Indosinian tectonic setting, magmatism and metallogenesis in Qinling Orogen, central China. Chin. Geol. 2010, 37, 854-865.

17. YU, C.; YU, J.-P. Structure-controlled Mineralization Rules and Their Implications to Prospecting in the Xialadi Lead-Zinc Deposit, Western Qinling. Geol. Explor. 2010, 46, 261-266.

18. Chen, Y.; Hu, R.; Bi, X.; Dong, S.; Xu, Y.; Zhou, T. Zircon U-Pb Ages and Sr-Nd-Hf Isotopic Characteristics of the Huichizi Granitic Complex in the North Qinling Orogenic Belt and Their Geological Significance. J. Earth Sci. 2018, 29, 492-507. [CrossRef]

19. Yuan, F.; Liu, J.; Carranza, E.J.M.; Zhai, D.; Wang, Y.; Zhang, S.; Sha, Y.; Liu, G.; Wu, J. The Guangshigou uranium deposit, northern Qinling Orogen, China: A product of assimilation-fractional crystallization of pegmatitic magma. Ore Geol. Rev. 2018, 99, 17-41. [CrossRef]

20. Zhu, L.; Zhang, G.; Yang, T.; Wang, F.; Gong, H. Geochronology, petrogenesis and tectonic implications of the Zhongchuan granitic pluton in the Western Qinling metallogenic belt, China. Geol. J. 2013, 48, 310-334. [CrossRef]

21. Qin, J.; Lai, S.; Grapes, R.; Diwu, C.; Ju, Y.; Li, Y. Geochemical evidence for origin of magma mixing for the Triassic monzonitic granite and its enclaves at Mishuling in the Qinling orogen (central China). Lithos 2009, 112, 259-276. [CrossRef]

22. Wang, T.; Ni, P.; Sun, W.; Zhao, K.; Wang, X. Zircon U-Pb ages of granites at Changba and Huangzhuguan in western Qinling and implications for source nature. Chin. Sci. Bull. 2011, 56, 659-669. [CrossRef]

23. Zhou, Z.-Y.; Yang, J.-X.; Tao, X.; Nie, L.-J.; Sun, L.-L. Geochronological and Geochemical Characteristics of Early Mesozoic Caochuanpu Granitoids in Tianshui Area of West Qinling and Their Geological Significance. J. Earth Sci. Environ. 2015, 37, 11-20.

24. Han, J.S.; Yao, J.M.; Chen, Y.J. Geochronology and geochemistry of the Dashui adakitic granitoids in the western Qinling Orogen, central China: Implications for Triassic tectonic setting. Geol. J. 2014, 49, 383-401. [CrossRef]

25. Li, N.; Chen, Y.-J.; Santosh, M.; Pirajno, F. Compositional polarity of Triassic granitoids in the Qinling Orogen, China: Implication for termination of the northernmost paleo-Tethys. Gondwana Res. 2015, 27, $244-257$. [CrossRef]

26. Wang, T.; Guo, L.; Zhang, L.; Yang, Q.; Zhang, J.; Tong, Y.; Ye, K. Timing and evolution of Jurassic-Cretaceous granitoid magmatisms in the Mongol-Okhotsk belt and adjacent areas, NE Asia: Implications for transition from contractional crustal thickening to extensional thinning and geodynamic settings. J. Asian Earth Sci. 2015, 97, 365-392. [CrossRef]

27. Kong, J.; Niu, Y.; Duan, M.; Zhang, Y.; Hu, Y.; Li, J.; Chen, S. Petrogenesis of Luchuba and Wuchaba granitoids in western Qinling: Geochronological and geochemical evidence. Mineral. Petrol. 2017, 111, 887-908. [CrossRef]

28. Zhu, L.-M.; Zhang, G.-W.; Chen, Y.-J.; Ding, Z.-J.; Guo, B.; Wang, F.; Lee, B. Zircon U-Pb ages and geochemistry of the Wenquan Mo-bearing granitioids in West Qinling, China: Constraints on the geodynamic setting for the newly discovered Wenquan Mo deposit. Ore Geol. Rev. 2011, 39, 46-62. [CrossRef]

29. Zhang, G.-W. Nature of main tectono-lithostratigraphic units of the Qinling orogen: Implications for the tectonic evolution. Acta Petrol. Sin. 1995, 11, 101-114. 
30. Dong, Y.; Zhang, G.; Hauzenberger, C.; Neubauer, F.; Yang, Z.; Liu, X. Palaeozoic tectonics and evolutionary history of the Qinling orogen: Evidence from geochemistry and geochronology of ophiolite and related volcanic rocks. Lithos 2011, 122, 39-56. [CrossRef]

31. Lü, T.-y.; Zhang, Y.-s.; Ruan, A.-g. The geological structure background and the crustal structure in the northeastern margin of the Qinghai-Tibetan plateau. Acta Seism. Sin. 2000, 13, 687-697.

32. Chen, Z.; Lu, S.; Li, H.; Li, H.; Xiang, Z.; Zhou, H.; Song, B. Constraining the role of the Qinling orogen in the assembly and break-up of Rodinia: Tectonic implications for Neoproterozoic granite occurrences. J. Asian Earth Sci. 2006, 28, 99-115. [CrossRef]

33. Chen, Y.J.; Santosh, M. Triassic tectonics and mineral systems in the Qinling Orogen, central China. Geol. J. 2014, 4, 338-358. [CrossRef]

34. Yu, X. Mantle xenoliths and megacrysts in ultramafic lamprophyres in Haoti, Gansu Province. Geol. Sci. Technol. Inf. 1991, 10, 97-108.

35. Yu, X.; Mo, X.; Cao, Y.; Dong, G. Cenozoic kamafugite volcanism and its petrological characteristics west Qinling, Gansu Province. In Proceedings of the Extended Abstract for the Second World Chinese Conference A, Stanford University, Stanford, CA, USA, 2-4 August 2000; pp. 249-254.

36. Yu, X. Cenozoic potassic alkaline ultrabasic volcanic rocks and its genesis in Lixian9Dangchang area, Gansu Province. Tethyan Geol. 1994, 18, 1149129.

37. Yu, X.; Zhao, Z.; Zhou, S.; Mo, X.; Zhu, D.; Wang, Y. ${ }^{40} \mathrm{Ar} /{ }^{39} \mathrm{Ar}$ dating for Cenozoic kamafugite from western Qinling in Gansu Province. Chin. Sci. Bull. 2006, 51, 1621-1627. [CrossRef]

38. Wang, B.; Wang, L.; Pan, G.; Yin, F.; Wang, D.; Tang, Y. U-Pb zircon dating of Early Paleozoic gabbro from the Nantinghe ophiolite in the Changning-Menglian suture zone and its geological implication. Chin. Sci. Bull. 2013, 58, 920-930. [CrossRef]

39. Diwu, C.; Sun, Y.; Zhao, Y.; Liu, B.; Lai, S. Geochronological, geochemical, and Nd-Hf isotopic studies of the Qinling Complex, central China: Implications for the evolutionary history of the North Qinling Orogenic Belt. Geosci. Front. 2014, 5, 499-513. [CrossRef]

40. Shi, Y.; Yu, J.-H.; Santosh, M. Tectonic evolution of the Qinling orogenic belt, Central China: New evidence from geochemical, zircon U-Pb geochronology and Hf isotopes. Precambrian Res. 2013, 231, 19-60. [CrossRef]

41. Nensong, C.; Yujing, H.; Zhendong, Y.; Min, S. Whole rock Sm-Nd, Rb-Sr and single grain zircon $\mathrm{Pb}-\mathrm{Pb}$ dating of complex rocks from the interior of the Qinling orogenic belt, western Henan, and its crustal evolution. Geochimica 1991, 3, 219-228.

42. Zhao, J.; Chen, D.; Tan, Q.; Chen, M.; Zhu, X.; Guo, C.; Liu, L. Zircon LA-ICP-MS U-Pb dating of basic volcanics from Erlangping Group of the North Qinling, eastern Qinling Mountains and its geological implications. Earth Sci. Front. 2012, 19, 118-125.

43. Liu, G.; Zhang, S.; You, Z. Metamorphic Rocks in Qinling Orogen Belt and Its Metamorphic Evolution; Geology Press: Beijing, China, 1993.

44. Yang, Z.; Chao, H.; Wu, X.; Su, S.; Yang, J.; Yang, R. Element evolution of the planet earth and the relative movement of the earth interior: Rediscuss on the new global geodynamic theory about the development and evolution of tectonics in Chin. Earth Sci. Front. 2016, 23, 166-182.

45. Gao, J. Analysis of trace elements in rock samples using HRICPMS. J. Nanjing Univ. (Nat. Sci.) 2003, 39, 844-850.

46. Gu, H.-O.; Xiao, Y.; Santosh, M.; Li, W.-Y.; Yang, X.; Pack, A.; Hou, Z. Spatial and temporal distribution of Mesozoic adakitic rocks along the Tan-Lu fault, Eastern China: Constraints on the initiation of lithospheric thinning. Lithos 2013, 177, 352-365. [CrossRef]

47. Wiedenbeck, M.; Hanchar, J.M.; Peck, W.H.; Sylvester, P.; Valley, J.; Whitehouse, M.; Kronz, A.; Morishita, Y.; Nasdala, L.; Fiebig, J. Further characterisation of the 91500 zircon crystal. Geostand. Geoanal. Res. 2004, 28, 9-39. [CrossRef]

48. Andersen, T. Correction of common lead in $\mathrm{U}-\mathrm{Pb}$ analyses that do not report 204Pb. Chem. Geol. 2002, 192, 59-79. [CrossRef]

49. Ludwig, K.R. User's manual for isoplot 3.00, a geochronlogical toolkit for microsoft excel. Berkeley Geochronl. Cent. Spec. Publ. 2003, 4, 25-32.

50. Streckeisen, A. Classification and nomenclature of plutonic rocks recommendations of the IUGS subcommission on the systematics of igneous rocks. Geol. Rundsch. 1974, 63, 773-786. [CrossRef] 
51. Middlemost, E.A. Magmas and Magmatic Rocks: An Introduction to Igneous Petrology; Addison-Wesley Longman Ltd.: Boston, MA, USA, 1985.

52. Chappel, B.; White, A. Two contrasting granite types. Pac. Geol. 1974, 8, 173-174.

53. White, A.J.; Chappell, B.W. Ultrametamorphism and granitoid genesis. Tectonophysics 1977, 43, 7-22. [CrossRef]

54. Chappell, B.W.; White, A. I- and S-type granites in the Lachlan Fold Belt. Earth Environ. Sci. Trans. R. Soc. Edinb. 1992, 83, 1-26.

55. Eby, G.N. The A-type granitoids: A review of their occurrence and chemical characteristics and speculations on their petrogenesis. Lithos 1990, 26, 115-134. [CrossRef]

56. Wright, J. A simple alkalinity ratio and its application to questions of non-orogenic granite genesis. Geol. Mag. 1969, 106, 370-384. [CrossRef]

57. Mackenzie, F.T.; Garrels, R. Evolution of Sedimentary Rocks; Norton: New York, NY, USA, 1971.

58. Frost, B.R.; Barnes, C.G.; Collins, W.J.; Arculus, R.J.; Ellis, D.J.; Frost, C.D. A geochemical classification for granitic rocks. J. Petrol. 2001, 42, 2033-2048. [CrossRef]

59. Pearce, J.A.; Harris, N.B.; Tindle, A.G. Trace element discrimination diagrams for the tectonic interpretation of granitic rocks. J. Petrol. 1984, 25, 956-983. [CrossRef]

60. Anders, E.; Grevesse, N. Abundances of the elements: Meteoritic and solar. Geochim. Cosmochim. Acta 1989, 53, 197-214. [CrossRef]

61. Rudnick, R.; Gao, S. Composition of the continental crust. Crust 2003, 3, 1-64.

62. Sun, S.-S.; McDonough, W.F. Chemical and isotopic systematics of oceanic basalts: Implications for mantle composition and processes. Geol. Soc. Lond. Spec. Publ. 1989, 42, 313-345. [CrossRef]

63. Bea, F.; Mazhari, A.; Montero, P.; Amini, S.; Ghalamghash, J. Zircon dating, Sr and Nd isotopes, and element geochemistry of the Khalifan pluton, NW Iran: Evidence for Variscan magmatism in a supposedly Cimmerian superterrane. J. Asian Earth Sci. 2011, 40, 172-179. [CrossRef]

64. Konzett, J.; Armstrong, R.A.; Sweeney, R.J.; Compston, W. The timing of MARID metasomatism in the Kaapvaal mantle: An ion probe study of zircons from MARID xenoliths. Earth Planet. Sci. Lett. 1998, 160, 133-145. [CrossRef]

65. Şengör, A. Geology: East Asian tectonic collage. Nature 1985, 318, 16-17. [CrossRef]

66. Liu, X.; Jahn, B.M.; Hu, J.; Li, S.; Liu, X.; Song, B. Metamorphic patterns and SHRIMP zircon ages of medium-to-high grade rocks from the Tongbai orogen, central China: Implications for multiple accretion/collision processes prior to terminal continental collision. J. Metamorph. Geol. 2011, 29, 979-1002. [CrossRef]

67. Zhang, H.-F.; Zhu, R.-X.; Santosh, M.; Ying, J.-F.; Su, B.-X.; Hu, Y. Episodic widespread magma underplating beneath the North China Craton in the Phanerozoic: Implications for craton destruction. Gondwana Res. 2013, 23, 95-107. [CrossRef]

68. Colman-Sadd, S. Two stage continental collision and plate driving forces. Tectonophysics 1982, 90, $263-282$. [CrossRef]

69. Hefferan, K.; Brien, J. Earth Materials; Wiley-Blackwell: Hoboken, NJ, USA, 2010.

70. Qin, J.; Lai, S.; Li, Y. Post-collisional plutonism with adakitic signatures: The Triassic Yangba granodiorite (Bikou terrane, northern Yangtze Block). Chin. J. Geochem. 2008, 27, 72. [CrossRef]

71. Vernon, R. Microgranitoid enclaves in granites-globules of hybrid magma quenched in a plutonic environment. Nature 1984, 309, 438-439. [CrossRef]

72. Baxter, S.; Feely, M. Magma mixing and mingling textures in granitoids: Examples from the Galway Granite, Connemara, Ireland. Mineral. Petrol. 2002, 76, 63-74. [CrossRef]

73. Qin, J.; Lai, S.; Li, Y. Slab breakoff model for the Triassic post-collisional adakitic granitoids in the Qinling Orogen, Central China: Zircon U-Pb ages, geochemistry, and Sr-Nd-Pb isotopic constraints. Int. Geol. Rev. 2008, 50, 1080-1104. [CrossRef]

74. Ellis, D.J.; Thompson, A.B. Subsolidus and partial melting reactions in the quartz-excess $\mathrm{CaO}+\mathrm{MgO}+$ $\mathrm{Al}_{2} \mathrm{O}_{3}+\mathrm{SiO}_{2}+\mathrm{H}_{2} \mathrm{O}$ system under water-excess and water-deficient conditions to $10 \mathrm{~kb}$ : Some implications for the origin of peraluminous melts from mafic rocks. J. Petrol. 1986, 27, 91-121. [CrossRef]

75. Douce, A.E.P. What do experiments tell us about the relative contributions of crust and mantle to the origin of granitic magmas? Geol. Soc. Lond. Spec. Publ. 1999, 168, 55-75. [CrossRef] 
76. Frost, B. Occurrence of irontitanium oxides in igneous rocks. Rev. Mineral. Oxide Miner. Petrol. Magn. Significance 1991, 25, 433-468.

77. Rapp, R.P.; Watson, E.B. Dehydration melting of metabasalt at 8-32 kbar: Implications for continental growth and crust-mantle recycling. J. Petrol. 1995, 36, 891-931. [CrossRef]

78. Smithies, R.; Champion, D. The Archaean high-Mg diorite suite: Links to tonalite-trondhjemite-granodiorite magmatism and implications for early Archaean crustal growth. J. Petrol. 2000, 41, 1653-1671. [CrossRef]

79. Liu, J.; Sun, Y.; Li, H.; Yang, L. LA-ICP-MS zircon dating of Sifangtai mafic-ultramafic complex in the North Qinling orogenic belt. Yanshi Kuangwuxue Zazhi (Acta Petrol. et Mineral.) 2012, 31, 524-530.

80. Nie, L. Study on Geochemical Characteristics and Zircon U-Pb Data of the Niutou River Granite Rock, Tianshui area. Master's Thesis, Chang'an University, Xi'an, China, 2014.

81. Condie, K.C.; Bowling, G.; Allen, P. Missing Eu anomaly and Archean high-grade granites. Geology 1985, 13, 633-636. [CrossRef]

82. Defant, M.J.; Drummond, M.S. Derivation of some modern arc magmas by melting of young subducted lithosphere. Nature 1990, 347, 662-665. [CrossRef]

83. Yang, H.; Zhang, F. Evolution and metallogenesis of the West Qinling Orogenic belt. Acta Geol. Sichuan 1996, $16,73-79$.

84. Wang, H.; Xu, X.; Chen, J.; Sun, Y.; Li, W.; Li, P.; Li, T.; Zhang, H. Dating and geochemical characteristics of the Yanwan Paleozoic collisional intrusion in the west segment of Northern Qinling Mts. Acta Geol. Sin. 2009, 83, 353-364.

85. Li, C.; Ren, L.; Zong, S.; Wang, Y.; Li, M. Confirmation of the Major Grenville Event in the Qinling Complex of the Qinling Orogenic Belt, Central China. J. Earth Sci. 2019, 30, 494-509. [CrossRef]

86. Zhang, J.; Yu, S.; Meng, F. Ployphase Early Paleozoic metamorphism in the northern Qinling orogenic belt. Acta Petrol. Sin. 2011, 27, 1179-1190.

87. Zhang, G.W. Formation and Evolution of the Qinling Orogen; Northwest University Press: Xi'an, China, 1988. (In Chinese)

88. Zhao, S.; Li, S.; Li, X.; Somerville, I.; Cao, H.; Liu, X.; Wang, P. Structural analysis of ductile shear zones in the North Qinling Orogen and its implications for the evolution of the Proto-Tethys Ocean. Geol. J. 2017, 52, 202-214. [CrossRef]

89. Lerch, M.; Xue, F.; Kröner, A.; Zhang, G.; Tod, W. A middle Silurian-Early Devonian magmatic arc in the Qinling Mountains of central China. J. Geol. 1995, 103, 437-449. [CrossRef]

90. Xue, F.; Kröner, A.; Reischmann, T.; Lerch, F. Palaeozoic pre-and post-collision calc-alkaline magmatism in the Qinling orogenic belt, central China, as documented by zircon ages on granitoid rocks. J. Geol. Soc. 1996, 153, 409-417. [CrossRef]

91. Pei, X.; Ding, S.; Li, Z.; Liu, Z.; Li, R.; Feng, J.; Sun, Y.; Zhang, Y.; Liu, Z.; Zhang, X. Early Paleozoic Tianshui-Wushan tectonic zone of the northern margin of West Qinling and its tectonic evolution. Acta Geol. Sin. 2009, 83, 1547-1564.

92. Chen, J.; Xu, X.; Wang, H.; Wang, Z.; Zeng, Z.; Wang, C.; Li, P. LA-ICPMS zircon U-Pb dating of Tangzang quartz-diorite pluton in the west segment of North Qinling Mountains and its tectonic significance. Geoscience 2008, 22, 63-70.

93. Liu, C.; Pei, X.; Li, Z.; Li, R.; Wang, Y.; Pei, L.; Chen, Y.; Wei, F.; Gao, J.; Wu, S. Moshigou meta-Basalt in the Tianshui area in the conjunction of the Qinling-Qilian orogens: Zircon U-Pb geochronology, geochemistry and tectonic implications. Acta Geol. Sin. 2014, 88, 347-360.

94. Dong, Y.; Genser, J.; Neubauer, F.; Zhang, G.; Liu, X.; Yang, Z.; Heberer, B. U-Pb and 40Ar/39Ar geochronological constraints on the exhumation history of the North Qinling terrane, China. Gondwana Res. 2011, 19, 881-893. [CrossRef]

95. Enkin, R.J.; Yang, Z.; Chen, Y.; Courtillot, V. Paleomagnetic constraints on the geodynamic history of the major blocks of China from the Permian to the present. J. Geophys. Res. Solid Earth 1992, 97, 13953-13989. [CrossRef]

96. Li, S.; Zhao, S.; Liu, X.; Cao, H.; Yu, S.; Li, X.; Somerville, I.; Yu, S.; Suo, Y. Closure of the Proto-Tethys Ocean and Early Paleozoic amalgamation of microcontinental blocks in East Asia. Earth-Sci. Rev. 2018, 186, 37-75. [CrossRef]

97. Francois, H.; Johannes, W. Genesis of peraluminous granites I. Experimental investigation of melt compositions at 3 and $5 \mathrm{~kb}$ and various $\mathrm{H}_{2} \mathrm{O}$ activities. J. Petrol. 1991, 32, 935-958. 
98. Pearce, J.A.; Peate, D.W. Tectonic implications of the composition of volcanic arc magmas. Annu. Rev. Earth Planet. Sci. 1995, 23, 251-285. [CrossRef]

99. Power, G. Chemical variation in tourmalines from south-west England. Mineral. Mag. J. Mineral. Soc. 1968, 36, 1078-1089. [CrossRef]

100. Charoy, B. Post-magmatic processes in south-west England and Brittany. Proc. Ussher Soc. 1981, 5, 101-115.

101. Charoy, B. Tourmalinisation in Cornwall, England. Met. Assoc. Magmat. 1982, 6, 63-70.

102. Floyd, P.A.; Exley, C.S.; Styles, M. The igneous rocks of south-west England. In Igneous Rocks of South-West England; Springer: Berlin/Heidelberg, Germany, 1993; pp. 1-7.

103. Ussher, W.A.E.; Barrow, G.; MacAlister, D.A. The Geology of the Country Around Bodmin and St. Austell; HM Stationery Office: Richmond, UK, 1909.

104. Chen, Y.J.; Santosh, M.; Somerville, I.; Chen, H.Y. Indosinian tectonics and mineral systems in China: An introduction. Geol. J. 2014, 49, 331-337. [CrossRef]

105. Huang, X.; Mo, X.; Yu, X.; Li, X.; Ding, Y.; Wei, P.; Wen, Y. Zircon U-Pb chronology, geochemistry of the Late Triassic acid volcanic rocks in Tanchang area, West Qinling and their geological significance. Acta Petrol. Sin. 2013, 29, 3968-3980.

106. Huang, W.; Wu, Z. Evolution of the Qinling orogenic belt. Tectonics 1992, 11, 371-380. [CrossRef]

107. Lai, S.; Zhang, G.; Yang, Y.; Chen, J. Geochemistry of the ophiolite and island arc volcanic rock in the MianxianPLueyang suture zone, southern Qinling and their tectonic significances. Geochimica 1998, 27, 283-293.

108. Li, N.; Chen, J.; Zhang, H. Molybdenum deposits in east Qinling. Earth Sci. Front. 2007, 14, 186.

109. Chen, Y.; Zhe, M.; Jiang, S. Significant achievements and open issues in study of orogenesis and metallogenesis surrounding the North China continent. Acta Petrol. Sin. 2009, 25, 2695-2726.

(C) 2020 by the authors. Licensee MDPI, Basel, Switzerland. This article is an open access article distributed under the terms and conditions of the Creative Commons Attribution (CC BY) license (http://creativecommons.org/licenses/by/4.0/). 